\title{
Wind Variability in BZ Camelopardalis
}

\author{
R.K. Honeycutt ${ }^{1}$, S. Kafka ${ }^{2}$, J.W. Robertson ${ }^{3}$
}

\begin{abstract}
Sequences of spectra of the nova-like cataclysmic variable (CV) BZ Cam were acquired on 9 nights in 2005-2006 in order to study the time development of episodes of wind activity known to occur frequently in this star. We confirm the results of Ringwald \& Naylor (1998) that the P-Cygni absorption components of the lines mostly evolve from higher expansion velocity to lower velocity as an episode progresses. We also commonly find blueshifted emission components in the $\mathrm{H} \alpha$ line profile, whose velocities and durations strongly suggest that they are also due to the the wind. Curiously, Ringwald \& Naylor reported common occurences of redshifted $\mathrm{H} \alpha$ emission components in their BZ Cam spectra. We have attributed these emission components in $\mathrm{H} \alpha$ to occasions when gas concentrations in the bipolar wind (both frontside and backside) become manifested as emission lines as they move beyond the disk's outer edge. We also suggest, based on changes in the P-Cygni profiles during an episode, that the progression from larger to smaller expansion velocities is due the higher velocity portions of a wind concentration moving beyond the edge of the continuum light of the disk first, leaving a net redward shift of the remaining absorption profile.

We derive a new orbital ephemeris for BZ Cam, using the radial velocity of the core of the HeI $5876 \AA$ line, finding $\mathrm{P}=0.15353(4)$. Using this period the wind episodes in BZ Cam are found to be concentrated near inferior conjuction of the emission line source. This result helps confirm that the winds in nova-like CVs are often phase dependent, in spite of the puzzling implication that such winds lack axisymmetry.

We argue that the radiation-driven wind in BZ Cam receives an initial boost by acting on gas that has been lifted above the disk by the interaction of the
\end{abstract}

\footnotetext{
${ }^{1}$ Astronomy Department, Indiana University, Swain Hall West, Bloomington, IN $47405 . \quad$ E-mail: honey@astro.indiana.edu

${ }^{2}$ Dept. of Terrestrial Magnetism, Carnegie Inst. of Washington, 5241 Broad Branch Road NW, Washington, DC 2001. E-mail: skafka@dtm.ciw.edu

${ }^{3}$ Arkansas Tech University, Dept. of Physical Sciences, 1701 N. Boulder Ave., Russellville, AR 72801-2222. E-mail: jrobertson@atu.edu
} 
accretion stream with the disk, thereby imposing flickering time scales onto the wind events, as well as leading to an orbital modulation of the wind due to the non-axisymmetric nature of the stream/disk interaction. Simultaneous photometry and spectroscopy were acquired on 3 nights in order to test the possible connection between flickering continuum light and the strength of the frontside wind. We found strong agreement on one night, some agreement on another, and no agreement on the third. We suggest that some flickering events lead to only backside winds which will not have associated P Cygni profiles.

Subject headings: stars:individual(BZ Cam)-cataclysmic variables-winds

\section{Introduction}

This is the first of an anticipated short series of papers on wind features in the optical spectra of nova-like (NL) cataclysmic variables (CVs). CV winds have mostly been studied at UV wavelengths accessible only from space. However, opportunities for UV spectroscopy from spacecraft have been restricted in recent years. Furthermore the time variability of the winds, which is the primary topic of this investigation, is more easily studied from the ground, where more numerous and longer sequences of spectroscopic and photometric monitoring are practical.

CVs are semi-detached interacting binary stars, in which the Roche-lobe-filling secondary star loses mass through its inner Lagrangian point to the white dwarf primary, forming an accretion disk (Warner 1995). The NL subset of CVs have higher mass transfer rates, resulting in a disk that is too hot for dwarf nova outbursts. In NL CVs seen at low orbital inclination, disk photons can be scattered in an expanding wind, resulting in blueshifted absorption line components (P-Cygni profiles) in lines such as CIV 1548-52. In high inclination (eclipsing) systems the line profile from the wind becomes purely broad emission near zero velocity. The general observational properties of CV winds have been summerized in Drew (1997)and in Froning (2005); wind models have been reviewed in Proga (2005). Maximum observed blueshifted absorption velocities reach $\sim 5000 \mathrm{~km} \mathrm{sec}^{-1}$. Estimated mass loss rates due to the wind vary considerably but values of $\dot{M}_{\text {wind }} \sim 10^{-2} \dot{M}$ of the accreted mass are not unusual. The loss of angular momentum via the wind may be an important fraction of the $\dot{L}$ needed to drive the mass transfer; see discussions in Cannizzo \& Pudritz (1988), Livio \& Pringle (1994), King \& Kolb (1995), and Knigge \& Drew (1997). The P Cygni lines are formed in a rotating bi-polar wind, but the mechanism for driving the wind remains somewhat uncertain. The most widely-accepted driving force is resonance scattering of photons from the hot accretion disk boundary layer, and/or hot white dwarf. 
Occasional P-Cygni profiles in the optical spectra of NL CVs have been known for some time, but recent work has shown line profiles due to winds to be relatively common in certain lines, at least for some stars. Kafka and Honeycutt (2004) discussed the use of the optical HeI lines and $\mathrm{H} \alpha$ to study the properties of disk winds in NL CVs. The orbital dependence and the secular time dependence are two important wind properties whose study is greatly eased, or made practical altogether, by the use of ground-based optical data. Examples of wind studies using these optical lines include Kafka et al. 2003 (Q Cyg), 2004 (BZ Cam, Q Cyg, HR Del, DI Lac, BT Mon, AT Cnc), and 2009 (V592 Cas). The HeI triplets $\left(2^{3} \mathrm{P}\right.$ $\rightarrow 3^{3} \mathrm{~S}$ and $2^{3} \mathrm{P} \rightarrow 3^{3} \mathrm{D}$ for the $5876 \AA$ and $7065 \AA$ lines respectively) are particularly windsensitive, showing P Cygni profiles when the wind is present, whereas the singlet He I line at $\lambda 6678$ arises primarily from the disk. Radiative transfer effects in HeI have been studied by Almog and Netzer (1989) and by Benjamin, Skillman \& Smits (2002). These lines have found particular application to the spectra of symbiotic stars (Proga, Mikolajewski \& Kenyon 1994; Silviero \& Munari 2003).

The strong metastability of the $2^{3} \mathrm{~S}$ level of He I (which is the effective ground state of the triplets) likely alters the recombination cascade in such a way as to make the triplets more liable to absorption under the low density conditions and dilute radiation field of the wind, compared to the singlet at $6678 \AA$. When P-Cygni profiles are present in the profiles of the HeI triplet lines, $\mathrm{H} \alpha$ also often shows wind features. Among numerous NL CVs which we have examnined for wind line profiles in the optical, BZ Cam is among the most active, having wind features about half the time, which is about the same rate found in optical spectroscopy of BZ Cam by Ringwald \& Naylor (1998; hereafter RN98). BZ Cam also has wind signatures in space-UV lines. (e.g. Hollis et al. 1992; Prinja et al. 2000).

The rapid variability of the BZ Cam wind has received past attention, including Griffith et al. (1995), Patterson et al.(1996), RN98, Prinja et al.(2000), and Greiner et al. (2001); episodic outflows have also been described in other NLs (e.g. Prinja et al. 2000 for V603 Aql; Prinja et al. 2003 for RW Sex). In general these studies describe wind events with lengths from 100 to $3000 \mathrm{sec}$, having blue-shifted absorption components reaching $-3000 \mathrm{~km} \mathrm{~s}^{-1}$, and little or no orbital dependence of the wind. RN98 report that the BZ Cam wind decelerates as an episode progresses, unlike the wind in OB stars. This present study incorporates optical spectroscopy of BZ Cam acquired during 2005-2006.. This is a considerably richer spectroscopic data set than earlier studies, allowing us to refine earlier results in the literature and also reveal additional wind phenonomena in BZ Cam. We also explore extensive longterm photometry of BZ Cam obtained 1990-2012.

BZ Cam is at a distance of $830 \pm 160 \mathrm{pc}$ (RN98). The energy distribution in the optical (Patterson et al. 1966) is quite blue, indicating a high $\dot{M}$ accretion system as is typical of 
most NL CVs which show winds. The orbital inclination is low, as judged by the lack of eclipses and the fact that the cores of the emission lines are quite narrow $(\sim 12 \AA \mathrm{FWHM})$. RN98 estimate an inclination of $12-40^{\circ}$.

BZ Cam is unusual for being the only CV embedded in a nebula that is not related to a recorded nova explosion. It has been suggested that interactions of the BZ Cam wind with the interstellar medium produces the observed nebular bow shock (Hollis et al. 1992), but alternative origins for the nebula have also been proposed (e.g. Griffith, Fabian \& Sion 1995; Greiner et al. 2001).

\section{Data Acquisition and Reductions}

\subsection{Photometry}

Table 1 is a $\log$ of the photometric observations. Column 1 provides a reference designation for each sequence, column 2 is a UT or UT range, while column 3 gives similar information for the JD. Column 4 is the telescope, column 5 the exposure time in sec, column 6 the number of useable exposures, and column 7 the duration of the data stream. Column 8 indicates which (if any) spectral sequence was simultaneous with the photometry. All of the photometry is in the $\mathrm{V}$ band.

Our long-term BZ Cam photometry, having typical spacings of days over intervals of many years, consists of three sets 1990-1996, 2000-2005, and 2007-2012, all using autonomous unattended telescopes in central Indiana. The first two such sequences (P1 and P2) were obtained using a 0.41-m telescope (informally called RoboScope; Honeycutt et al. 1994 and references therein). Flats and other detector calibration data were automatically acquired and applied each night, followed by aperture photometry and field identification, all using custom software (Honeycutt \& Turner 1992). Final photometric reductions were done using the incomplete ensemble technique contained in Astrovar, which is a custom package based on the technique described in Honeycutt (1992), but with the addition of a graphical user interface. The RoboScope Astrovar solution used 32 ensemble stars, and the zeropoint was established to within $0.01 \mathrm{mag}$ using 10 secondary standards from Henden \& Honeycutt (1995). The third long-term sequence (P3) was acquired 2007-2012 using an unattended, autonomous 1.25-m telescope at the same site as RoboScope. The P3 data were reduced using a custom pipeline consisting of IRAF1 routines for detector calibrations, followed by the

\footnotetext{
${ }^{1}$ IRAF is distributed by the National Optical Astronomy Observatories, which are operated by the Association of Universities for Research in Astronomy, Inc., under cooperative agreement with the National
} 
application of SExtractor 2 for aperture photometry. The light curves were then generated using Astrovar, employing a total of 24 ensemble comparison stars. The zero point was determined to within $0.01 \mathrm{mag}$ using 11 secondary standards from Henden \& Honeycutt (1995). Sequence P3 is missing data between 2009-Apr-25 and 2010-Oct-07 (the full 20092010 winter observing season for BZ Cam) because of a detector failure.

Sequences P4, P5, and P6 are short-term photometry having typical spacings of minutes over a single night each. Data for P4 are from the Tenagra Observatory $30.8-\mathrm{m}$ telescope in southern Arizona and the data for Sequences P5 and P6 are from the 0.91-m WIYN 4 telescope at Kitt Peak. The purpose of these sequences was to provide photometry simultaneous with spectroscopic sequences. Both the Tenagra and WIYN 0.91-m exposures were reduced using differential photometry from C-Munipack 5 , a PC (Windows)-based photometry package orginally developed by Hroch (1998) and maintained by David Motl. This processing included detector calibrations as well as aperture photometry. The differential photometry was placed on the standard system using comparison star HH6 (V=14.00) from Henden \& Honeycutt (1995). Errors were assigned using the check star HH02 (V=13.38)

Figure 1 shows our full light curve made up of all the sequences in Table 1. There are unfortunate gaps in the Indiana long-term coverage, especially 1996-2000. Compilation of averages of visual estimates in the AAVSO archives do not begin until 2000-Dec and therefore do not help much with filling this gap. During the small intervals of overlap the AAVSO visual estimates and the Indiana data agree reasonably well (to within $\sim 0.1 \mathrm{mag}$ ), except for a number of isolated faint AAVSO visual estimates at $\mathrm{V}=14.2-15.6$ between 2007-Mar and 2009-Apr. There are no similar faint data points in the relatively complete Indiana data over this same time interval, so it will be important to learn if such faint states actually occur in BZ Cam. We note that Garnavich \& Szkody (1988) found a 1928 low state of BZ Cam at V=14.1, using the Harvard Plate Collection. Also shown in Figure 1 are our straight line characterizations of the light curve from Greiner et al. (2001), whose data are from a variety of sources but mostly VSNET. During the interval of overlap from JD 2451780 to 2451870 the data in the Greiner et al. compilation are $~ 0.35$ mag brighter than

Science Foundation.

${ }^{2}$ SEextractor is a source detection and photomery package described by Bertin and Arnouts 1996. It is available from http://terapix.iap.fr/soft/sextractor/.

${ }^{3}$ http://www.tenagraobservatories.com/

${ }^{4}$ The WIYN Observatory is a joint facility of the University of Wisconsin-Madison, Indiana University, Yale University, and the National Optical Astronomy Observatory

5 http://C-Munipack.sourceforge.net 
the Indiana photometry. During this same interval the Indiana photometry agrees with the AAVSO visual estimates to $\sim 0.1 \mathrm{mag}$.

Table 2 lists all the JDs, magnitudes, and errors (with respect to the ensemble) of the new Indiana data. The complete version of Table 2 is available only in electronic form. Errors (not shown in Figure 1 but included in Table 1) are for differential magnitudes with respect to the ensemble, and are mostly $\sim 0.02 \mathrm{mag}$.

\subsection{Spectroscopy}

Our time-resolved spectroscopic data were obtained using a variety of telescopes during the interval 2005-Oct to 2006-Sep. The spectroscopic sequences totaled 26 hours on 9 nights. The wavelength regions varied somewhat but always included the four lines HeI $5876 \AA$, H $\alpha$, HeI $6678 \AA$, and HeI $7065 \AA$.

Table 3 is a journal of our spectroscopic runs. Column 1 assigns a designation to each spectral sequence for subsequent reference, while columns 2 and 3 give the UT date and the JD. Column 4 is the telescope, column 5 is the exposure time in sec, and column 6 is the number of useable exposures in the sequence. Column 7 gives the duration of the sequence in hours, column 8 indicates if simulaneous photometry is available (see Table 1), and column 9 notes whether the sky was clear, mostly clear, or partly cloudy. Figure 2 is an expanded portion of the BZ Cam light curve of Figure 1, with the times of our spectra marked.

Spectral sets S1, S2, and S3 were obtained using the GoldCam slit spectrograph on the KPNO6 2.1-m telescope. Grating 35 was used in first order, providing coverage $~ 5400-7400 \AA$ at $\sim 3 \AA$ resolution. Spectral sets S4, S7, and S8 were obtained using the MOS/Hydra multiple object spectrograph on the WIYN 7 telescope. Grating 600 was used in first order, providing coverage $\sim 5300-8200 \AA$. The "red" 2 " fiber bundle was employed, yielding $\sim 3 \AA$ resolution; numerous other fibers were used for sky subtraction. Spectral sets S5 and S6 were obtained using the RC slit spectrograph on the Kitt Peak 4-m telescope. Grating KPC-007 was used in first order, providing coverage $\sim 5600-7900 \AA$ at $\sim 3 \AA$ resolution. Spectral set S9 used the

\footnotetext{
${ }^{6}$ Kitt Peak National Observatory is a division of the National Optical Astronomy Observatory, which is operated by the Association of Universities for Research in Astronomy, Inc., under cooperative agreement with the National Science Foundation

${ }^{7}$ The WIYN Observatory is a joint facility of the University of Wisconsin-Madison, Indiana University, Yale University, and the National Optical Astronomy Observatory.
} 
6.5-m MMT8 at Mt. Hopkins, Arizona. The Blue Channel slit spectrograph was employed with a 1200 line/mm grating, providing coverage $\sim 5800-7100 \AA$ at a resolution of $\sim 1.5 \AA$.

For all spectra the detector calibrations used standard IRAF 9 procedures, and for spectral extractions and wavelength calibrations we used IRAF's onedspec/twodspec packages. No spectrophotometric calibrations were applied for any of the spectra, and the continua in the reduced spectra were nomalized to unity.

\section{Qualitative Results and Comparisons with Earlier Work}

For NL CVs prone to wind activity, it has been found (Kafka \& Honeycutt 2004) that the HeI triplet lines at $5876 \AA$ and $7065 \AA$ often display wind features in their line profiles, while the HeI singlet at $6678 \AA$ never shows wind features. This behavior is fully confirmed in our BZ Cam spectroscopy. Because HeI 6678 is uninteresting insofar as wind studies, and because the HeI $7065 \AA$ line profile seems to simply be a weaker (and therefore noiser) version of the HeI $5876 \AA$ profile, we have concentrated our attention on just two lines: HeI $5876 \AA$ and $\mathrm{H} \alpha$.

The line profiles for the HeI 5876 line and for $\mathrm{H} \alpha$ are displayed in two different complementary ways. In Figure 2 we show nested plots of the line profiles, while in Figure 3 we show the same profiles as simulated trailed spectra with the intensities coded as scales of grey. The nested plots are best for revealing relative strengths and shapes of wind features, while the trailed spectra are better for showing the time dependence of the line profiles. The two presentations use the same data. However, the trailed spectra have been rather strongly contrast-enhanced to emphasize the time development of the wind episodes. We encourage the reader to use both sets of figures as we describe the variablity in the BZ Cam wind. Due to clouds, the spectra in sequences S6, S7, and S8 are sometimes quite noisy. We nevertheless have included them in our analysis because the wind features are still visible, and the information provided on duty cycles and time dependences remain valuable to our study.

The P-Cygni profiles seen in Figures 2 and 3 occur in short bursts of activity, with durations ranging from 7 to $90 \mathrm{~min}$. The mean is $\sim 45 \mathrm{~min}$. Because the duty cycle of the

\footnotetext{
${ }^{8}$ The MMT Obervatory is a joint facility of the Smithsonian Institution and the University of Arizona

${ }^{9}$ IRAF is distributed by the National Optical Astronomy Observatories, which are operated by the Association of Universities for Research in Astronomy, Inc., under cooperative agreement with the National Science Foundation.
} 
episodes is near $50 \%$, some of the longer duration episodes probably are overlapping shorter episodes. The mode of the distribution of episode durations ( $\sim 35 \mathrm{~min})$ may therefore be more meaningful. These episode durations are consistent with that found by RN98 of $\sim 40$ min for spectra on two nights in 1995 over 7.5 total hours. It is likely that our exposure times undersample some of the more rapid changes. In fact, Prinja et al. (2000) reported wind variability times scales down to $100 \mathrm{sec}$ in HST UV spectra of BZ Cam. Nevertheless, except at the start of the wind episodes, there are no discontinuities in the line profiles between adjacent spectra in Figures 2 and 3, leading us to conclude that undersampling is not a serious problem for our data.

As seen in Figure 1, BZ Cam was fairly stable in brightness 1991-96, followed by erratic variations in the range $\mathrm{V}=12-14$ during $1997-2012$, on time scales of weeks to months. Although photometric coverage near the times of our spectra is spotty, it appears from examination of Figure 1 that our spectra were acquired when the system was near $\mathrm{V}=13.3$, which would be an intermediate (or even intermediate/faint) state. The fact that the BZ Cam wind is quite active in our spectra is at variance with the conclusion of Greiner et al. (2001) that the BZ Cam wind shows up only in the high state and is absent during the optical low and intermediate states. It may be that the BZ Cam wind varies on many time scales, some of which have yet to be sampled.

As can be seen in Figure 3, the episodes of P Cygni absorption seem to begin abruptly and simultaneously in HeI 5876 and in $\mathrm{H} \alpha$, and the evolution of the profiles during an event are similar for two lines. In most of the wind episodes the blueshifted absorption evolves towards slower expansion velocity as the event progresses. Although they start at the same time, the blueshifted absorption components of the line profiles typically persist for $\sim 2 \times$ longer in $\mathrm{HeI}$ than in $\mathrm{H} \alpha$.

RN98 reported a blueward linear increase in velocity for the first 6-8 min of a wind episode in BZ Cam, followed by a deceleration to near rest in 30-40 min. We see a rather wider range of behavior with no evidence for an acceleration phase. However, our results may be consistent RM98 when one recognizes that our spectral sequences would barely resolve a 6-8 min velocity increase to begin the wind event (RN98 had $60 \mathrm{~s}$ time resolution). As best seen in Figure 2, the blue absorption in our spectra typically begins as a broad, shallow feature, which becomes deeper as the event progresses, as well as moving redward in mean velocity as the bluer portions of the absorption disappear. This happens in both HeI and $\mathrm{H} \alpha$ but is easier to see in HeI because the events last longer and the absorption is often stronger. Other changes sometimes occur in the shape of the blueshifted absorption as the event progresses, such as development of sawtoothed profiles or multiple components (e.g. profiles 3-50, 3-51, 4-09, 4-10, 5-10, 5-12.) These more complicated profiles are nevertheless 
usually superimposed on the general trend from broad shallow absorption to more narrow, deeper, less blueshifted profiles as the event progresses. The largest blueshifted absorption velocities are $\sim-2350 \mathrm{~km} \mathrm{~s}^{-1}$, compared to maximum velocities near $-3000 \mathrm{~km} \mathrm{~s}^{-1}$ in RN98.

As best seen in Figure 3 the $\mathrm{H} \alpha$ line profile often shows a blueshifted emission component, which is never seen in HeI. The velocities of the blueshifted $\mathrm{H} \alpha$ emission ranges up to $-2200 \mathrm{~km} \mathrm{~s}^{-1}$, similar to that of the blueshifted absorption in HeI $5876 \AA$ and in $\mathrm{H} \alpha$. We do not see much systematic velocity evolution of the blueshifted $\mathrm{H} \alpha$ emission during a wind event. The velocities of the emission in most of sequences appears random; however, there is some evolution towards lower velocities in sequences $\mathrm{S} 2$ and S5. The blueshifted $\mathrm{H} \alpha$ emission seems to often come after the appearance of blueshifted absorption in HeI and in $\mathrm{H} \alpha$. However, the time relationship of these events is difficult to pin down in our data sets because the typical length of our spectral sequences not much longer than the typical spacing between events, and because wind events sometimes overlap. Because the time relationship of the various wind manifestions is important to our interpretations, we will return to this point in later discussion.

RN98 report occasional blueshifted $\mathrm{H} \alpha$ emission in BZ Cam spectra, but the appearences were relatively brief, weak, and much less conspicious than in our BZ Cam spectra. RN98 also saw frequent and conspicious redshifted $\mathrm{H} \alpha$ emission in their BZ Cam spectra, reaching up to $+2400 \mathrm{~km} \mathrm{~s}^{-1}$ (which can also be seen in the spectra of Patterson et al. 1996). Curiously we do not see redshifted $\mathrm{H} \alpha$ emission in any of our BZ Cam spectra reported here (though we did see redshifted $\mathrm{H} \alpha$ emission in a single BZ Cam spectrum acquired in 2004 (Kafka \& Honeycutt 2004)). Both the redshifted and blueshifted $\mathrm{H} \alpha$ emission features are surely due to the wind, because they have velocities and time scales similar to the P Cygni absorption features.

The wind features seen in Sequence S9 (the MMT spectra) are different from the behaviors seen in the other sequences. In S9 the blue absorption in HeI is present nearly all the time, mostly at the same relatively low velocity. There is a short wind episode near the end of the Sequence S9 having the characteristic decline from high to low expansion velocities. However, in HeI this episode is weak and not well-defined. The $\mathrm{H} \alpha$ profile shows broad weak absorption at high negative velocities for the first $2 / 3$ of the sequence, followed by a brief wind episode at the end of the sequence, whose timing and velocities mirror that seen in HeI episode. There is occasional absorption in the red wing of $\mathrm{H} \alpha$, for which a physical explanation is lacking. Furthermore that feature (all well as a similar absorption feature in the blue wing of $\mathrm{HeI}$ ) seem to vary with a quasi-periodicity of 8-9 min (see Fig 3e). (However, when the residual intensities in the relevant spectral windows were plotted vs. time, no meaningful quasi-periodicities were apparent.) Sequence S9 has the most favorable 
combination of spectral resolution and $\mathrm{S} / \mathrm{N}$ of our 9 sequences, so it is possible that these relatively weak behaviors are also present in the other sequences but are simply not apparent in spectra having lower $\mathrm{S} / \mathrm{N}$ and/or lower spectral resolution. Because the S9 features are at variance with those in S1-S8, we considered whether unknown data acquisition or data reduction problems could be responsible. However, such features are lacking in the spectra of other CVs acquired on the same night, and we are inclined to regard the odd line profiles in the S9 spectra as real.

\subsection{Orbital Ephemeris}

A reliable orbital ephermeris for BZ Cam is desired for two purposes: 1) to correct the spectra for orbital motion before extracting velocities of the wind features, and 2) to investigate any possible dependence of the wind parameters on orbital phase. We expected that our fairly extensive spectroscopy (9 nights, well-distributed over a year, and having 4 emission lines) would allow determination of an accurate and reliable ephemeris. To minimize wind contributions we measured the radial velocities of the central emission cores of $\mathrm{H} \alpha$, HeI $5876 \AA$, HeI $6678 \AA$, and HeI $7065 \AA$ in all our spectra. (HeI $7065 \AA$ was sometimes too faint for reliable measurement.) However, preliminary periodogram analysis of these velocities proved discouraging. Systematic radial velocity variations with typical full amplitudes of $\sim 150 \mathrm{~km} \mathrm{~s}^{-1}$ are present for most of the lines for most of the runs in Table 1, all having characteristic "periods" near $3.7 \mathrm{hr}$. However, periodograms using the full year of data seldom show significant peaks. One exception is the periodogram for HeI 5876 which has a significant peak near 0.1535 days. This peak is consistent with the spectroscopic period of Lu and Hutchings (1985) of $0.1535 \mathrm{~d}$ (using 49 spectra over 134 days) and with the Patterson et al. (1996) spectroscopic period of 0.1533(3) d (using 28 spectra over 5 successive nights). However, it is inconsistent with the Patterson et al. photometric period of 0.153693(7). (This photometric period used extensive photometry of BZ Cam over $\sim 4$ months in 1994-95, and nearby aliases were addressed by appealing to the radial velocity data of Lu \& Hutchings (1985) as well as to the possible spectroscopic periods in the Patterson et al. spectra. Patterson et al. suggest that their photometric period might be affected by superhumps.) Unfortunately the $0.1535 \mathrm{~d}$ period lacks sufficient accuracy for use over our one year of 20052006 spectroscopy, and the photometric period of Patterson et al. (1996) does not phase our radial velocity data at all.

Upon folding the He 5876 radial velocity curve on this initial period it was obvious that much of the difficulty was due to a varying gamma velocity from night to night. To address this deficiency we "pre-whitened" the data by adjusting the velocites for each night 
to each have an average of zero velocity. This is an imperfect process because many of the 9 spectral sequences cover less than a full orbit, causing the mean velocity to depend on the particular phases that were covered. Nevertheless this prewhitening dramatically increased the reliability of the detected period and resulted in a folded radial velocity curve of reasonably good quality (Figure 4). The resulting ephemeris for the times of gamma crossing from - to + velocity for the HeI 5876 emission core is

$$
\mathrm{T}_{0}=2453654.008(2)+0.15353(4)^{*} \mathrm{E} .
$$

This folded radial velocity curve has $\mathrm{K}=84(5) \mathrm{km} \mathrm{s}^{-1}$. This new ephemeris has sufficient accuracy to phase our spectroscopy over one year, and is the one we will use in this paper. Assuming that HeI 5876 emission arises from the vicinity of the mass-gaining star (a reasonable assumption considering the strong wind profile seen in this line), then phase zero corresponds to superior conjunction of the mass-losing star.

Our new period also appears in a periodogram (the periodgrams are not shown) of the radial velocities of the $\mathrm{H} \alpha$ emission core, at about the same accuracy and sharpness as in HeI 5876, but it is only the fourth strongest peak in the interval 0.1 to 0.2 days. This is in contrast to the periodograms of He I 5876, where this period is the strongest in the interval 0.1-0.2 days, for both the raw and the prewhitened radial velocities. One of the reasons that the peak is missing or weak in some lines can be seen in Figure 5. In this figure we compare, as an example, the radial velocity curves for four spectral lines from Sequence S5. We see that the velocity curves of HeI 5876 and HeI 6678 resemble one another, but that HeI 7065 and $\mathrm{H} \alpha$ are nearly antiphased to that of $\lambda 5876$ and $\lambda 6678$. The singlet HeI 6678 line seems to never display P-Cygni profiles, unlike either $\mathrm{H} \alpha$ or the two triplet He I lines ( $\lambda 5876$ and $\lambda 7065$ ); this holds true in both in BZ Cam and in other CV winds (Kafka \& Honeycutt 2004 and refs therein). We might therefore expect the $\lambda 6678$ radial velocity curve to be the least affected by the wind and to be the "odd man out" among the lines, but that is not the case. Spectral sequences other than S5 show similar discordant behaviors, but less pronounced.

Faced with such divergent behaviors among the radial velocity curves of different lines at the same epoch, we choose to use the He I 5876 ephemeris which (at least when prewhitened) does give a good period that is consistent with earlier work. We note that the three Balmer lines used in Lu \& Hutchings (1985) were also found to sometimes give differing velocities, and similar effects are present in the radial velocities of Patterson et al. 1996) data as well. We suspect that the wind is subtly distorting the emission line profiles in BZ Cam even in the cores of the lines, and even when P Cygni profiles are not apparent. We conjecture that this distortion differs from line-to-line, much as the P Cygni profiles differ from line-to-line, giving rise to discordant r.v. curves for different lines. For poorly understood reasons the core of HeI 5876 does give consistent r.v. results for our epochs, providing the additional 
decimal place in the period that is needed to phase our data over one year.

\subsection{Quantitative Characterization of the Wind Evolution}

We tried fitting multiple components to line profiles, but the profiles are so complicated that our results were quite dependent on the number of components chosen. The fact that blueshifted $\mathrm{H} \alpha$ emission sometimes overlaps blueshifted $\mathrm{H} \alpha$ absorption further complicated the fitting. Therefore we chose instead the use of direct summations to extract quantitative measures of the strengths and velocities from the line profiles, which does not require assuming anything about the profile shapes or the number of components. We defined a window between between -750 and $-2500 \mathrm{~km} \mathrm{~s}^{-1}$ (in the center-of-mass rest frame) over which to perform numerical integrations. The blue edge of the window was chosen so as to capture the bluest portions of the profile features due to the wind, while the red edge was fixed so as to try to avoid the strong central emission. (Because the central emission line and the wind features sometimes overlap, the separation is not perfect, but is largely effective). All pixel values above the continuum were assumed to belong the emission component, and those below to absorption. These integrations were converted to equivalent widths (EW), which were taken as measures of the emission and absorption strengths, and the first moments of the distributions were taken as the characteristic wavelength (i.e., velocity) of the absorption and emission features. That is,

$$
\begin{aligned}
& E W_{e m}=\Sigma\left(1-I_{i}\right) \Delta \lambda, \text { summed over the window, for } I_{i}>1, \\
& E W_{a b s}=\Sigma\left(1-I_{i}\right) \Delta \lambda, \text { summed over the window, for } I_{i}<1, \\
& \lambda_{e m}=\Sigma \lambda_{i}\left(I_{i}-1\right) / \Sigma \lambda_{i}, \text { summed over the window, for } I_{i}>1, \text { and } \\
& \lambda_{a b s}=\Sigma \lambda_{i}\left(1-I_{i}\right) / \Sigma \lambda_{i}, \text { summed over the windown, for } I_{i}<1 .
\end{aligned}
$$

Note that both absorption and emission are measured from the same wavelength window. Also note that because the continuum will have some noise, there will always be some pixels above the continuum, leading to a small but finite emission line EW even in the absence of an emission feature, with a corresponding effect in absorption.

Figure 6 shows the results of these measures for each of the nine sequences, where we have plotted vs. time the r.v. and EW of the blueshifted absorption component of both HeI 5876 and $\mathrm{H} \alpha$ (top two panels). The bottom panel plots the EW vs time for the blueshifted emission of $\mathrm{H} \alpha$. We have not plotted the velocities of the blueshifted emission of $\mathrm{H} \alpha$ because the feature is usually very broad with mostly random mean velocity changes. Also, velocities are not plotted when the line is weak and near the detection limit. The errors on the EWs 
and velocities can be judged by the scatter in the plots during times when the feature is not varying or (in the case of the EWs) when the feature is at or near zero. EW errors are typically $0.1-0.3 \AA$ and the r.v. errors are typically $100-300 \mathrm{~km} \mathrm{~s}^{-1}$

Examining the middle panels of Figure 6 we see that, in general, the velocities of the wind absorption decrease with time, approximately linearily. For 10 relatively isolated blueshifted absorption events in the top panels of Figure 6, which are also strong enough to have radial velocity measurements in the middle panels of Figure 6, we find 3 events with weak or illdefined velocity evolution, 1 event in which the absorption becomes bluer, and 6 events for which the absorption evolves towards lower expansion velocities. RN98 also concluded that most BZ Cam wind events decelerated with time. This trait is therefore a frequent and persistent property of the wind episodes in BZ Cam, but contrary behavior is sometimes seen.

\subsection{Orbital Dependence of the Wind}

The EW of the blueshifted HeI 5876 absorption appears to be the most sensitive and reliable indicator of wind activity in BZ Cam. It is generally stronger than the blueshifted $\mathrm{H} \alpha$ aborption and, unlike $\mathrm{H} \alpha$, is not contaminated by overlapping emission from the wind. Using the HeI EWs shown in the top panels of Figure 6 we have plotted these measures of wind strength vs. orbital phase in Figure 7. Data points belonging to same spectral sequence are connected by straight lines, showing that the episodic nature of the wind occurs at all orbital phases. Nevertheless the episodes are clearly concentrated to phases near or just following superior conjunction of the mass-losing star.

\subsection{Simultaneous Photometry and Spectroscopy}

On three nights we were able to obtain simultaneous $\mathrm{V}$ band photometry to accompany the spectroscopy. Figure 8 compares the V-band brightness of the system to the strength of the wind, as measured by the EW of blueshifted absorption in HI 5876. In the top panel it appears that all of the undulations are in common to the continuum brightness and to the strength of the wind. (It is unfortunate that the strong wind event at the end was missed in the photometry.) The middle panel shows no correlation between the photometry and the wind. In the bottom panel the slow rise over the middle portion of the observing window is in common to the photometry and to the wind strength, but there are no photometric features corresponding to the wind enhancements at the beginning and end of the sequence. 


\section{Discussion}

RN98 discuss how the apparent deceleration during a BZ Cam wind event is (surprisingly) opposite to the acceleration seen in the winds in OB stars, whose line profiles resemble those of NL CVs. They suggest that the effect may be due to wind dilution as it expands. We think that the apparent slowing of the wind velocities in BZ Cam is a geometrical effect due to the component of motion of the absorbing wind across the accretion disk as a wind event progresses. Imagine that a wind event involves the ejection of one (or a few) blobs of gas having a range of speeds, at a moderate opening angle. This will produce a range of transverse velocities of the absorbing wind blobs, such that those wind components with larger transverse velocity will complete their motion across the face of the disk before those components with smaller transverse velocity. Those faster components will also produce greater blueshifted absorption as long as the wind remains projected in front of the continuum source of the disk. Our evidence for this scenario is that the line profile changes seen during the velocity decline are such that the high velocity absorption disappears first, leaving only low velocity absorption late in the event. That is, the profile change is not one in which a given range of absorption velocities shift together to the red, but rather one in which the selective removal of the most blueshifted absorption as the event progresses leads to a net redward shift. At our time resolution the wind events seem to almost always begin with a very broad shallow absorption, which evolves to profiles having deeper, more narrow, and less blueshifted absorption. This systematic change in line profile can be seen in most of the wind episodes displayed in Figure 2, when the $\mathrm{S} / \mathrm{N}$ is favorable. For example, note the He I 5876 line profile changes in spectra 1-01 to 1-08, 3-01 to 3-06, 3-50 to 3-59, 4-09 to 4-16, and 5-09 to 5-27. Typically the velocity has declined in $\sim 40 \mathrm{~min}$ before disappearing due to weakness and/or merger with the central emission line. Following RN98, a disk radius of $4 \times 10^{10} \mathrm{~cm}$ (characteristic of luminous CVs) will be traversed in $40 \mathrm{~min}$ for a transverse velocity of $170 \mathrm{~km} \mathrm{~s}^{-1}$. Considering the uncertainties in the wind opening angle and the fraction of the disk traversed during a wind event, this rough agreement seems reasonable.

In the scenario just outlined, the blueshifted emission components of $\mathrm{H} \alpha$ seen in some of our spectra might result from gas that has passed beyond the edge of the disk continuum source, thereby changing from absorption to emission. If this interpretation of the nature of the blueshifted $\mathrm{H} \alpha$ emission components is correct, then we would expect to find that blueshifted $\mathrm{H} \alpha$ emission components systematically appear after a P-Cygni absorbing episode in HeI 5876. Unfortunately our spectral sequences are not long enough or extensive enough for a definitive test, but we can nevertheless make a quantitative consistency check. There is an interval following the start of a HeI 5876 P Cygni absorption episode during which we have the opportunity to check for blueshifted $\mathrm{H} \alpha$ emission that might result from the late stages of the wind event. This window of opportunity (WOO) may be terminated by either 
the beginning of new wind episode, or by the termination of the spectral sequence. The typical spacing between wind episodes is only $\sim 80 \mathrm{~min}$, which provides only a few useable WOO's having well-defined pairs of HeI blueshifted absorption events and blushifted $\mathrm{H} \alpha$ emission events. In Figure 6b (spectral sequence S2) we find a delay of 29 min between the start of the HeI 5876 absorption and the start of the blueshifted $\mathrm{H} \alpha$ emission, within a WOO of $172 \mathrm{~min}$. Therefore the blueshifted $\mathrm{H} \alpha$ emission occurs after a fraction 0.16 of the TOO has elapsed. There are two such pairings in Figure 6e (spectral sequence S5), yielding ratios Delay/WOO of $36 / 115=0.31$ and $43 / 94=0.46$. Because our sample is so small, a probability discussion is not appropriate. Instead we simply note that, compared to a random distribution of blushifted $\mathrm{H} \alpha$ emission within a TOO, all 3 occurances are early in the TOO, consistent with our hypothesis. Furthermore, the measured delays are consistent with estimates of disk transit times.

Similarly, the redshifted $\mathrm{H} \alpha$ emission components in the RN98 spectra might be due to wind concentrations on the backside of the disk, after they have passed out from behind the disk. In that case the passage of a backside wind blob across the disk does not produce a P Cygni aborption event, so we expect no correlation of HeI 5876 absorption with redshifted $\mathrm{H} \alpha$ emission. We attempted an analysis of the RN98 results in a fashion similar to that just described for the blueshifted $\mathrm{H} \alpha$ components in our spectra. However, difficulties with small numbers of useable pairings rendered this attempt futile. We do note that RN98 concluded that the red emission wing of $\mathrm{H} \alpha$ was uncorrelated with the $\mathrm{P}$ Cygni events in their data, which is consistent with our suggested scenario.

There have been numerous tests for an orbital dependence of the wind in NL CVs using space UV lines, with mixed results. This work has been hampered by various combinations of limited S/N, limited time resolution, and limited number of orbits. The limited number of orbits is a particular handicap for those systems in which the finite length of wind episodes can mimic a dependence on orbital phase. Taken together these studies (listed in Prinja et al. 2004 and in Froning 2005) demonstrate that orbit-modulated winds do occur, in spite of the difficulty in interpretation. The FUSE observations of the wind in V592 Cas (Prinja et al. 2004) is a study in which the orbital modulation is very secure. Furthermore this study was able to rule a wind modulation on either the positive or negative superhump period in V592 Cas, implying that neither disk eccentricity nor tilt is responsible for the orbital modulation. The orbital modulation of the wind in RW Sex (Prinja et al. 2003) provides further evidence that phase-dependent variations in $\mathrm{CV}$ winds are not tied to disk tilt or to disk eccentricity, because SW Sex is not a superhump system. Insofar as tests for an orbital dependence of the wind in BZ Cam itself, the IUE study of Griffith, Fabian \& Sion (1995) provided only a suggestion of such an effect, while the HST UV spectral study of Prinja et al. (2000) did not find any conclusive evidence for phase modulated absorption changes 
in the wind. Optical tests for the phase dependence of the winds in NL CVs are rare, but Kafka et al. (2009) found that the wind events in V592 Cas seemed to be concentrated near phase zero ( - to + crossing of $\gamma$ for the HeI emission lines, or superior conjuction of the secondary star). This is the same phase range in which we find the BZ Cam wind episodes to be concentrated.

Prinja et al. (2004) discuss the puzzle of an outflow modulated on the orbital period, reviewing the observations and suggested solutions. After ruling out eccentric or tilted disks, one of the few mechanisms remaining as possibilities for breaking the axisymmetry of the wind in V592 Cas (and by implication in the winds of NL CVs as well) is effects arising where the accretion stream meets the disk at a particular disk azimuth. Stream impact velocities are $\sim 5-10 \times$ smaller than wind velocities, so it is unlikely that the stream/disk interaction can produce a wind directly. Instead, we propose that stream impact and penetration raises gas clumps far enough above the plane of the disk that radiation pressure from inner disk can accelerate these clumps to the observed outflow velocities. In this scenario the stream/disk impact serves to imprint the observed episode time scales onto the wind (from the larger amplitude portion of the flickering), and also produces an orbital modulation.

Time dependent models of radiation driven winds from luminous accretion disks (Proga, Stone \& Drew 1998) produce stochastic velocity and density fluctuations in the wind with characteristic time scales of a few hundred minutes, if the radiation field arises mostly from the disk. The cause is the difference in the variation with height of the vertical components of gravity and radiation force. The fluctuating, clumpy, turbulant layer is concentrated near the plane of the disk, at low expansion velocities, whereas the BZ Cam wind variations encompass a wide range of velocities. However, the time scales of the variations produced by these models are intrigingly similar to the wind episode time scales seen in BZ Cam. The wind variablility mechanisms described in Proga et al (1998) may well be in play for CV winds like those in BZ Cam, even if other mechanisms (such as the hotspot-assisted radiation driven wind we are proposing) leads to the the observational effects discussed in this paper. Note that the Proga et al. models have cylindrical symmetry and therefore cannot address the orbital modulation of the wind, which is integral to our suggested scenario.

In Figure 7 we see that the strongest blueshifted wind absorption occurs near orbital phase zero, or superior conjunction of the secondary star. If the stream/disk interaction is responsible for launching the localized density enhancements that become the wind episodes, then does this orbital phase make sense? In high inclination CVs a "hump" in continuum light often appears just before inferior conjunction of the secondary star, arising from the "face-on" presentation of the impact point of the accretion stream with the accretion disk. (Actually, while hot spot humps are common in high inclination dwarf nova CVs they are 
rare in NL CVs such as BZ Cam, a complication we can ignore for now.) In high inclination NL CVs there is often evidence of stream/disk interactions on the far side of the disk, which can happen when the stream is thicker than the disk and overflows the initial impact point. This overflow can re-impact the disk on the far side, producing an interaction site that is at a quite different disk azimuth than the original disk impact azimuth, and often much nearer to the inner disk. These effects have been explored theoretically in studies such as Lubow (1989) and Armitage \& Livio $(1996 ; 1998)$. They have also been studied observationally, mostly in the context of the SW Sex phenomenon (e.g. Szkody \& Piché 1990; Hellier \& Robinson 1994; Knigge et al. 1994; Hoard et al. 1998). In general, these studies have found that the second stream/disk interaction can take place at a variety of disk azimuths and radii which are well inside the outer edge of the disk and mostly on the far side of the disk from the initial stream impact point. Therefore we do not think that the phase of maximum wind strength in Figure 27 is particularly significant, especially since in this low inclination system the structures are mostly visible at all orbital phases. Models of stream/disk interaction sites by Armitage \& Livio (1996) can produce a "spray" of debris above the disk. It was speculated that this gas may break up into clouds under the influence of radiation from the central source (or clumps may be imposed by irregularities in the stream). We speculate that such clumps are then accelerated by radiation pressure, imposing the flickering time scales on the wind episodes as well introducing departures from axisymmetry in the wind. In BZ Cam the flickering power extends to frequencies as low as the typical wind episode intervals (Patterson et al. 1996). Therefore a comparison of the $\mathrm{V}$ magnitude to the wind episodes bears on the feasibilty of the scenario just proposed.

Figure 8 shows that system brightness (V magnitude) and wind strength (as measured by the EW of blueshifted absorption in HeI 5876) are sometimes, but not always, correlated with one another. The data in the top panel shows good time correlation between the two measures. In the region of overlap nearly all of the inflections are in common to both the wind and the flickering brightness. In the middle panel there is no correlation between wind strength and flickering, while in the bottom panel there is some agreement over the midrange, but the two wind episodes have no counterpart in the photometry. Overall it is seen that the timescales for the wind changes and the flickering are quite similar, even if not always well correlated. It is easy to imagine that complexities in the details of the stream/disk interaction could result in some flickering events primarily producing only backside wind events, leaving no P Cygni signature. This speculation might account for the disappointing degree of correlation in the bottom two panels of Figure 28.

The process we have outlined (a hot spot assist to a radiation-driven wind) adds an additional mechanism to the usual scenario for wind generation. Nevertheless we think that a confrontation with Occam's razor is not unfavorable, because the proposed scenario 
addresses two additional observational properties: 1) the episodic nature of the winds in NL CVs, and 2) the orbital dependence of the wind. This proposed scheme is schematic and qualitative at this point, and will need additional observations and modeling (which are outside the scope of this paper) to establish its true merit.

\section{Summary and Conclusions}

Our primary observational results and suggested interpretations can be summerized as follows:

1) The wind episodes in BZ Cam typically last 35 min, during which a systematic decline of the velocities of the blushifted absorption occurs. This decline is attributed to the motion of wind concentrations across the face of the disk, whereby the blobs producing the largest blueshifts are more rapidly lost from the absorption line profile as they move beyond the source of background continuum light from the disk.

2) We find numerous examples of high velocity blueshifted emission components to the $\mathrm{H} \alpha$ line profile, which we attribute to emission from blobs of wind on the frontside of the disk after the gas concentrations have passed the outer edge of the disk. These features are in contrast to similar high velocity redshifted emission components in the $\mathrm{H} \alpha$ line profile found in RN98, which may be due to analogous backside wind events. We fnd that the delays between the start of a wind episode and the appearance of a $\mathrm{H} \alpha$ emission component is consistent with this scenario, but this result is not definitive because of small sample sizes.

3) We provide a new orbital ephemeris for BZ Cam, using the central core of the HI 5876 line. This ephemeris has sufficient accuracy to correct our line profiles for orbital motion and to reveal that the BZ Cam wind events are concentrated near the time of superior conjunction of the secondary star. We find that the orbital variations of the emission line radial velocities are inconsistent from line-to-line, and only the core of HeI 5876 gives good results.

4) Simultaneous photometric and spectroscopic sequences on three nights found that the flickering was well correlated with the wind on one night, somewhat correlated on a second, and uncorrelated on a third night. The timescales for the flickering and for the wind variability were similar.

5) Because the flickering is (at least sometimes) correlated with the wind, and the wind events are concentrated at a particular orbital phase, we suggest that the BZ Cam wind is generated by radiation pressure acting on gas blobs that are initially raised above the disk by 
the interaction of the accretion stream with the disk. In this way the stream/disk interaction

can imprint upon the wind both the observed flickering time scales and the observed orbital dependence of the wind episodes.

\section{Acknowledgments}

We are happy to acknowledge useful conversations with Daniel Proga regarding models of $\mathrm{CV}$ winds, and particularly of his time-dependent wind models.

\section{REFERENCES}

Almog, Y. \& Netzer, H. 1989, MNRAS, 238, 57

Armitage, P.J. \& Livio, M. 1996, ApJ, 470, 1024

Armitage, P.J. \& Livio, M. 1998, ApJ, 493, 898

Benjamin, R.A., Skillman, E.D. \& Smits, D.P. 2002, ApJ, 569, 288

Bertin, E., \& Arnouts, S. 1996, A\&AS, 117, 393

Cannizzo, J.K. \& Pudritz, R.E. 1988, ApJ, 327, 840

Drew, J.E. 1997, in Accretion Phenomena and Related Outflows, ASP Conf. Series 121, ed. D.T. Wickramasinghe, L. Ferrario \& G.V. Bicknell, p. 465

Froning, C.S. 2005, in Cataclysmic Variables and Related Objects, ASP Conf. Series 330, ed. J.-M. Hameury \& J.-P. Lasota, p. 81

Garnavich, P. \& Szkody, P. 1988, PASP, 100, 1522

Greiner, J. Tovmassian, G., Orio, M., Lehmann, H. Chavushyan, V., Rau, A., Schwarz, R., Casalegno, R.M \& Scholz, R.-D 2001, A\&A, 376, 1031

Griffith, D., Fabian, D. \& Sion, E.M. 1995, PASP, 107, 856

Hellier, C. \& Robinson, E.L. 1994, ApJ, 431, L107

Henden, A. A., \& Honeycutt, R. K. 1995, PASP, 107, 324

Hoard, D.W., Still, M.D., Szkody, P., Smith, R.C. \& Buckley, D.A.H. 1998, MNRAS, 295, 689

Hollis, J.M., Oliversen, R.J., Wagner, R.M. \& Feibelman, W.A. 1992, ApJ, 393, 217

Honeycutt R. K. 1992, PASP, 104, 435 
Honeycutt, R.K. \& Turner, G.W. 1992, in Robotic Telescopes in the 1990's, ed. A.V. Filippenko, ASP Conf Series 34, 77

Honeycutt, R.K., Adams, B.R., Swearingen, D.J. \& Kopp, W.R. 1994, PASP, 106, 670

Honeycutt, R.K. \& Kafka, S. 2004, AJ, 128, 1279

Hroch, F. 1998, Computer Programs for CCD Photometry, Proc. 29th Conf. on Var. Star Research, Nov. 1997, Brno, Czech Republic, ed. J. Dusek \& M. Zejda, 30

Kafka, S., Tappert, C., Honeycutt, R.K. \& Bianchini, A.2003, AJ,126, 1472

Kafka, S., Tappert, C., Honeycutt, R.K. \& Bianchini, A. 2004, RevMexAA, 20, 237

Kafka, S. \& Honeycutt, R.K. 2004, AJ, 128, 2420

Kafka, S., Hoard, D.W., Honeycutt, R.K. \& Deliyannis, C.P. 2009, AJ, 137, 197

King, A.R. \& Kolb, U. 1995, ApJ, 439, 330

Knigge, C., Drew, J.E., Hoare, M.G. \& la Dous, C. 1994, MNRAS, 269, 891

Knigge, C. \& Drew, J.E. 1997, ApJ, 486, 445

Livio, M. \& Pringle, J.E. 1994, ApJ, 427, 956

Lu, W., \& Hutchings, J. B. 1985, PASP, 97, 990

Lubow, S.H. 1989, ApJ, 340, 1064

Patterson, J., Patino, R., Throstensen, J.R., HarD., Skillman, D.R. \& Ringwald, F.A. 1996, AJ, 111, 2422

Prinja, R.K., Ringwald, F.A., Wade, R.A. \& Knigge, C. 2000, MNRAS, 312, 316

Prinja, R.K., Long, K.S., Froning, C.S., Knigge, C., Witherick, D.K., Clark, J.S. \& Ringwald, F.A. 2003, MNRAS, 340, 551

Prinja, R.K., Knigge, C., Witherick, D.K., Long, K.S. \& Brammer, G. 2004, MNRAS, 355, 137

Proga, D., Stone, J.M. \& Drew, J.E. 1998, MNRAS, 295, 595

Proga, D., Mikolajewska, J. \& Kenyon, S.J. 1994, MNRAS, 268, 213

Proga, D. 2005, in Cataclysmic Variables and Related Objects, ASP Conf. Series 330, ed. J.-M. Hameury \& J.-P. Lasota, p. 103

Ringwald, F.A. \& Naylor, T. 1998 (RN98), AJ, 115, 286

Siviero, A. \& Munari, U. 2003, in ASP Conf Series 303, ed. R.L.M Corradi, J. Mikolajewska \& T.J. Mahoney, p. 167

Szkody, P. \& Piché, F. 1990, ApJ, 361, 235 
Warner, B. 1995, Cataclysmic Variable Starrs (Cambridge:Cambridge Univ. Press)

This preprint was prepared with the AAS LATEX macros v5.2. 
Table 1. Photometry Log

\begin{tabular}{|c|c|c|c|c|c|c|c|}
\hline Sequence & $\mathrm{UT}$ & JD & $\mathrm{Tel} / \mathrm{Obs}$ & Secs & \# Exps & Dur. & Simul. Sp. \\
\hline $\mathrm{P} 1$ & $\begin{array}{l}\text { 1990-May-16 to } \\
1996-O c t-14\end{array}$ & $\begin{array}{l}2448027 \text { to } \\
2450370\end{array}$ & IU $0.41-\mathrm{m}$ & 120 & 489 & $6.4 \mathrm{yr}$ & \\
\hline $\mathrm{P} 2$ & $\begin{array}{l}2000-\text { Aug- } 15 \text { to } \\
2005-\text { Feb-21 }\end{array}$ & $\begin{array}{l}2451771 \text { to } \\
2453432\end{array}$ & IU 0.41-m & 120 & 508 & $4.5 \mathrm{yr}$ & \\
\hline P3 & $\begin{array}{l}\text { 2007-Sep-01 to } \\
\text { 2012-Apr-09 }\end{array}$ & $\begin{array}{l}2454344 \text { to } \\
2456036\end{array}$ & IU $1.25-\mathrm{m}$ & 90 & 269 & $4.6 \mathrm{yr}$ & \\
\hline $\mathrm{P} 4$ & 2005-Oct-12 & 2453655 & Tenagra 0.76-m & 90 & & $3.5 \mathrm{hr}$ & S3 \\
\hline P5 & 2006-Feb-21 & 2453787 & WIYN 0.91-m & 60 & 126 & $5.4 \mathrm{hr}$ & $\mathrm{S} 7$ \\
\hline $\mathrm{P} 6$ & 2006-Feb-23 & 2453789 & WIYN 0.91-m & 60 & 115 & $4.6 \mathrm{hr}$ & $\mathrm{S} 8$ \\
\hline
\end{tabular}


Table 2. Magnitudes for BZ Cam

\begin{tabular}{cccc}
\hline \hline JD & V Mag & Error & Source \\
\hline 2448207.73917 & 12.453 & 0.022 & IU $0.41-\mathrm{m}$ \\
2448207.95582 & 12.509 & 0.012 & IU $0.41-\mathrm{m}$ \\
2448208.82453 & 12.471 & 0.011 & IU $0.41-\mathrm{m}$ \\
2448209.68741 & 12.386 & 0.019 & IU $0.41-\mathrm{m}$ \\
2448233.74992 & 12.614 & 0.019 & IU $0.41-\mathrm{m}$ \\
2448234.63977 & 12.445 & 0.013 & IU $0.41-\mathrm{m}$ \\
2448234.81600 & 12.629 & 0.024 & IU $0.41-\mathrm{m}$ \\
2448235.61472 & 12.471 & 0.015 & IU $0.41-\mathrm{m}$ \\
2448235.96588 & 12.492 & 0.010 & IU $0.41-\mathrm{m}$ \\
$\ldots \ldots \ldots \ldots .$. & $\ldots \ldots$. & $\ldots \ldots$ & $\ldots \ldots$ \\
$\ldots \ldots \ldots \ldots .$. & $\ldots \ldots$. & $\ldots \ldots$ & $\ldots .$. \\
$\ldots \ldots \ldots \ldots .$. & $\ldots \ldots$ & $\ldots \ldots$ & $\ldots \ldots$ \\
2456027.59723 & 13.050 & 0.004 & IU $1.25-\mathrm{m}$ \\
2456029.61310 & 12.720 & 0.004 & IU $1.25-\mathrm{m}$ \\
2456036.58051 & 13.042 & 0.003 & IU $1.25-\mathrm{m}$ \\
\hline
\end{tabular}


Table 3. Spectroscopy Log

\begin{tabular}{ccccccccc}
\hline \hline \multirow{2}{*}{ Sequence } & UT & \multirow{2}{*}{ JD } & Tel & Secs & \# Exps & Hrs & Phot? & Clouds \\
\hline S1 & 2005-Oct-10 & 2453653 & KPNO 2.1-m & 300 & 8 & 0.8 & & clear \\
S2 & 2005-Oct-11 & 2453654 & KPNO 2.1-m & 180 & 40 & 2.6 & m. clear \\
S3 & 2005-Oct-12 & 2453655 & KPNO 2.1-m & 180 & 59 & 3.9 & P4 & clear \\
S4 & 2005-Oct-25 & 2453668 & WIYN 3.5-m & 300 & 17 & 2.2 & & clear \\
S5 & 2006-Jan-01 & 2453736 & KPNO 4.0-m & $120-180$ & 69 & 3.8 & clear \\
S6 & 2006-Jan-03 & 2453738 & KPNO 4.0-m & $180-600$ & 22 & 3.3 & p. cldy \\
S7 & 2006-Feb-21 & 2453787 & WIYN 3.5-m & 300 & 35 & 4.2 & P5 & p. cldy \\
S8 & 2006-Feb-23 & 2453789 & WIYN 3.5-m & 300 & 26 & 3.5 & P6 & p. cldy \\
S9 & 2006-Sep-15 & 2453993 & MMT 6.5-m & 90 & 40 & 1.3 & & clear \\
\hline
\end{tabular}




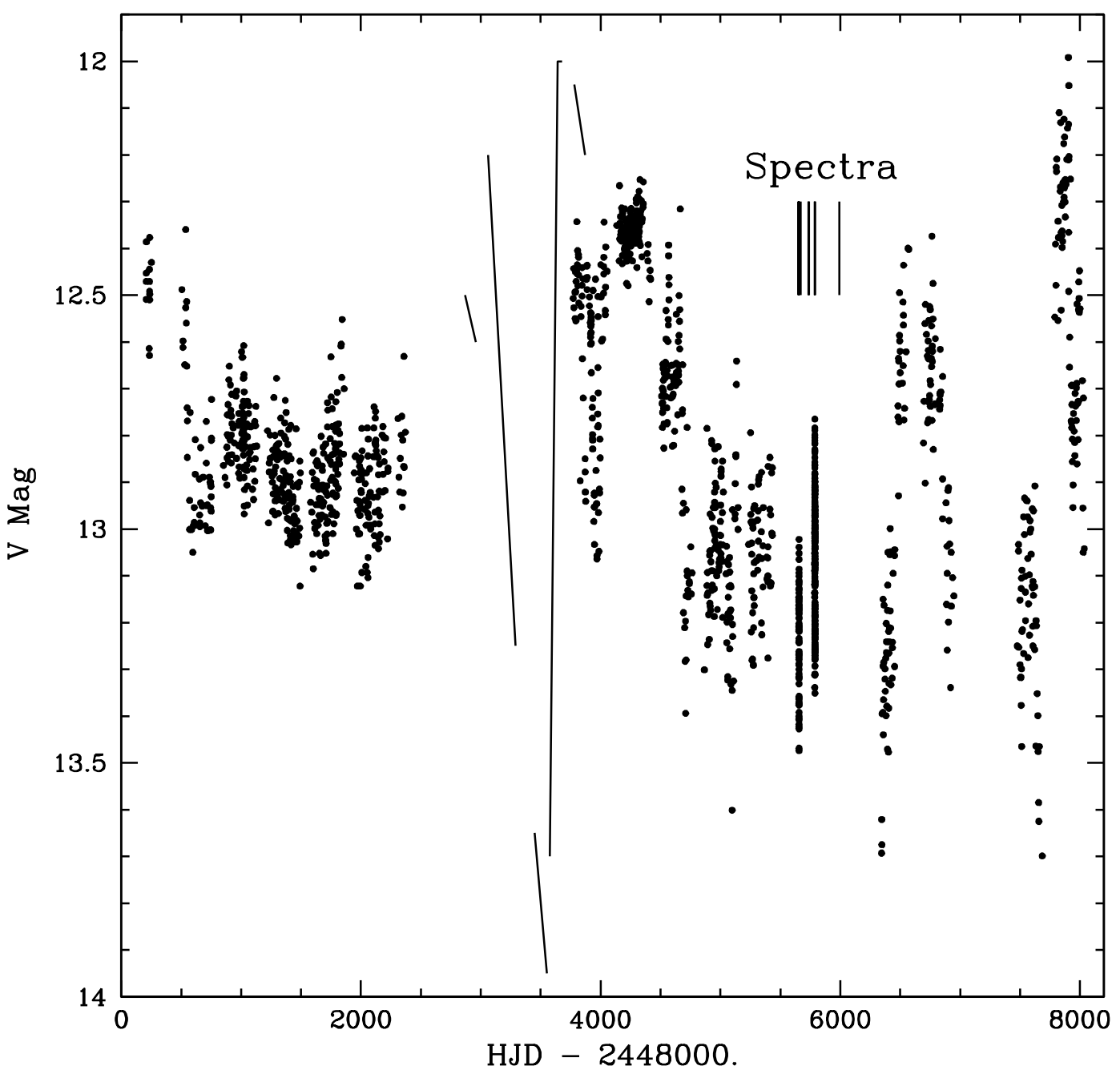

Fig. 1.- The full light curve of BZ Cam from 1990-Nov-12 to 2012-Apr-09. The vertical clusters of unresolved points are continuous sequences of exposures during a night. In the region 3000-3800 on the JD scale, where we have no original data, we have added as solid straight lines our characterization of the BZ Cam photometry found in Greiner et al. (2001), which were compiled from a variety of sources. The times of the 9 spectral sequences are marked with vertical lines. 

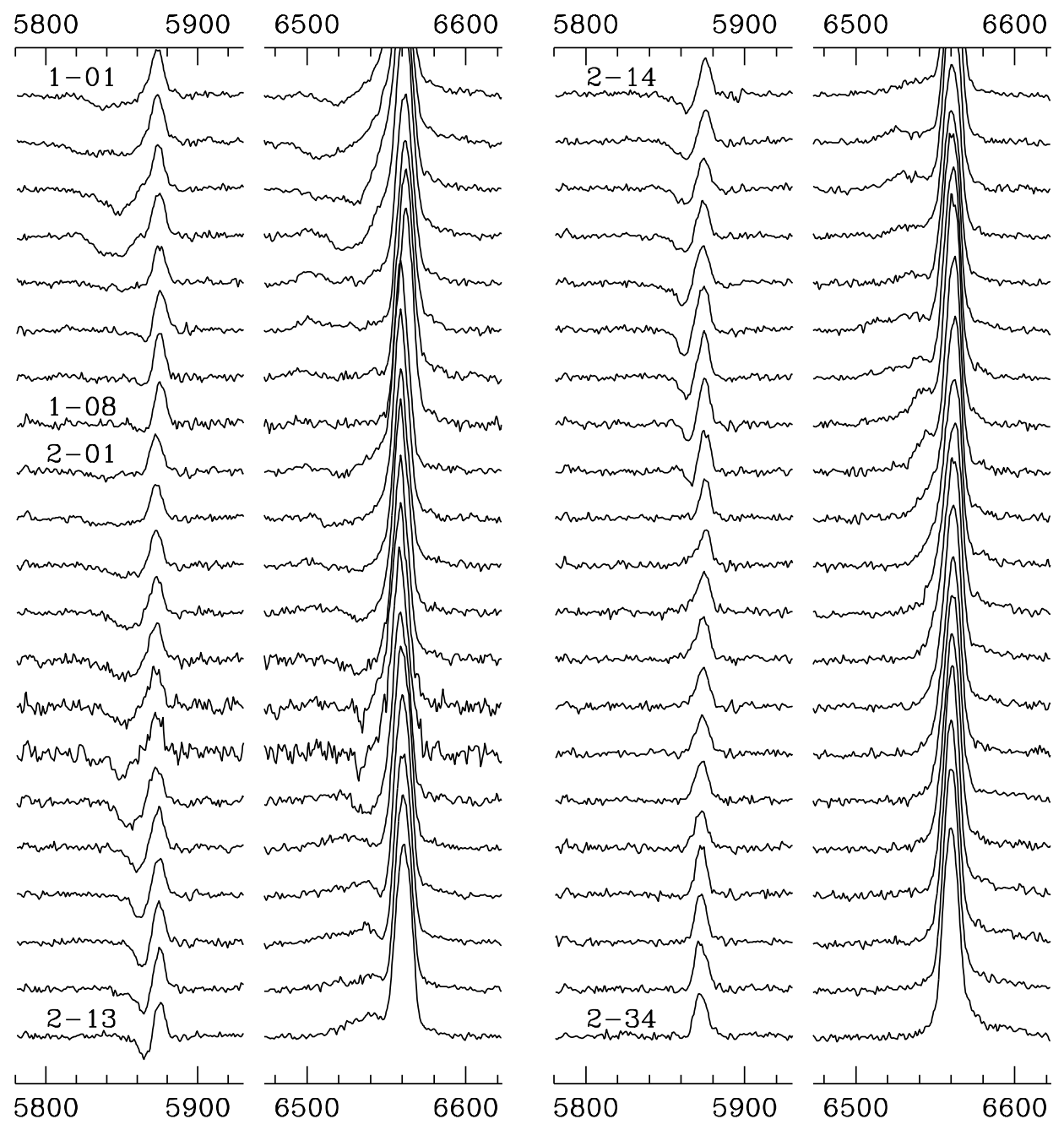

Fig. 2a.- Nested plots of the line profiles of HeI 5876 and $\mathrm{H} \alpha$. Time runs down the page. A profile is labeled if it begins/ends a column or begin/ends a sequence. 


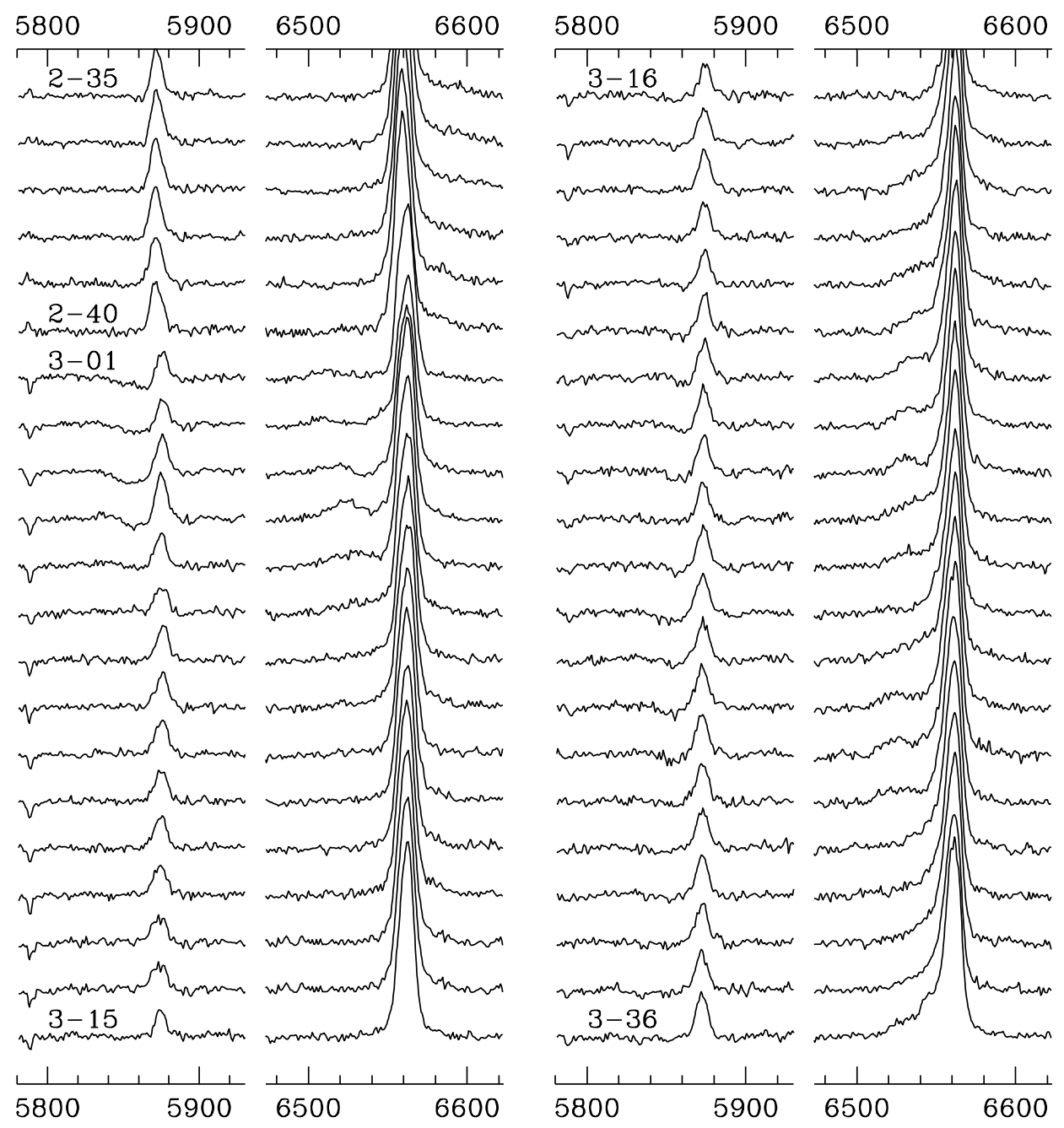

Fig. 2b.- Continuation of the line profile plots. 


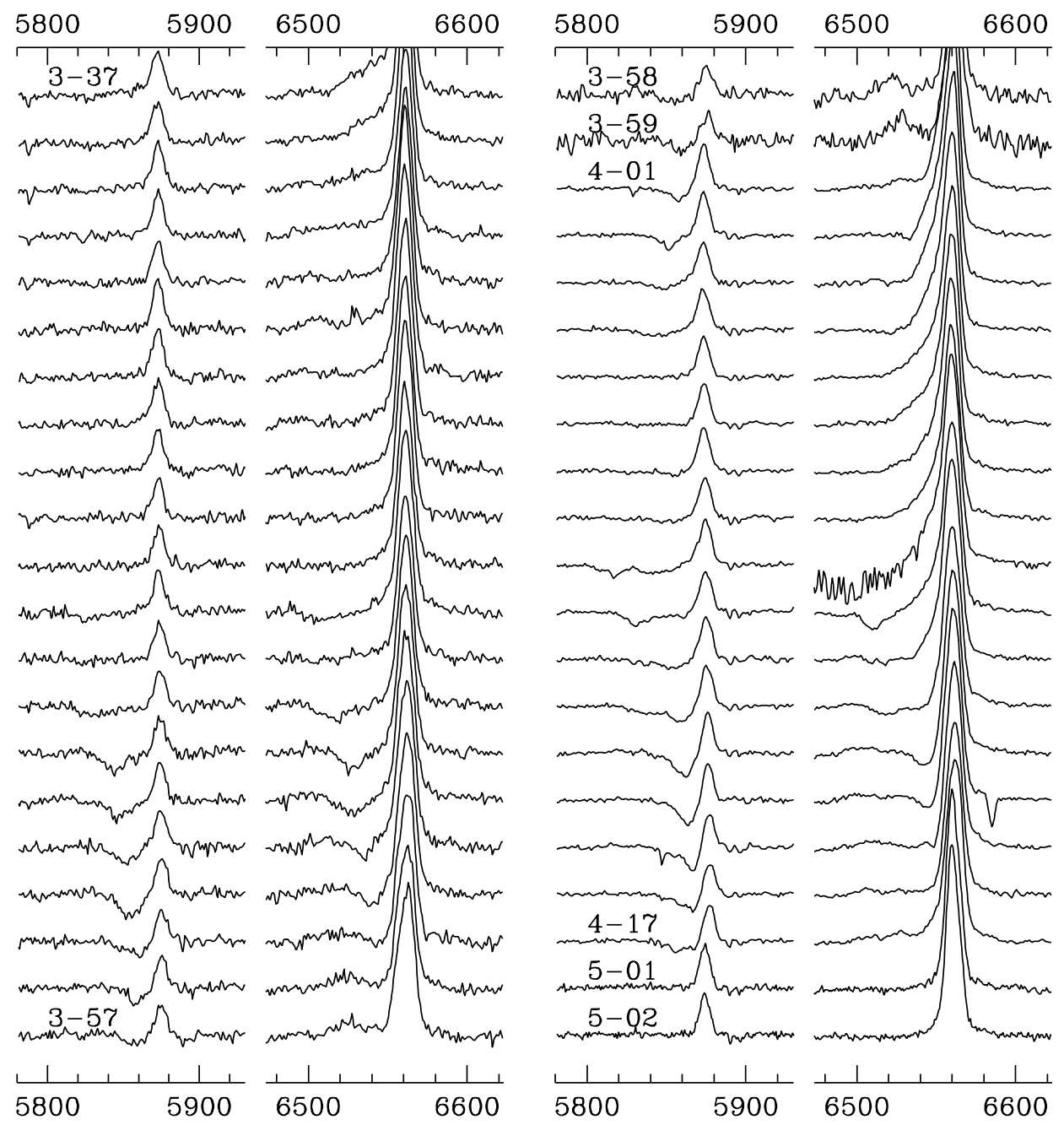

Fig. 2c.- Continuation of the line profile plots. 


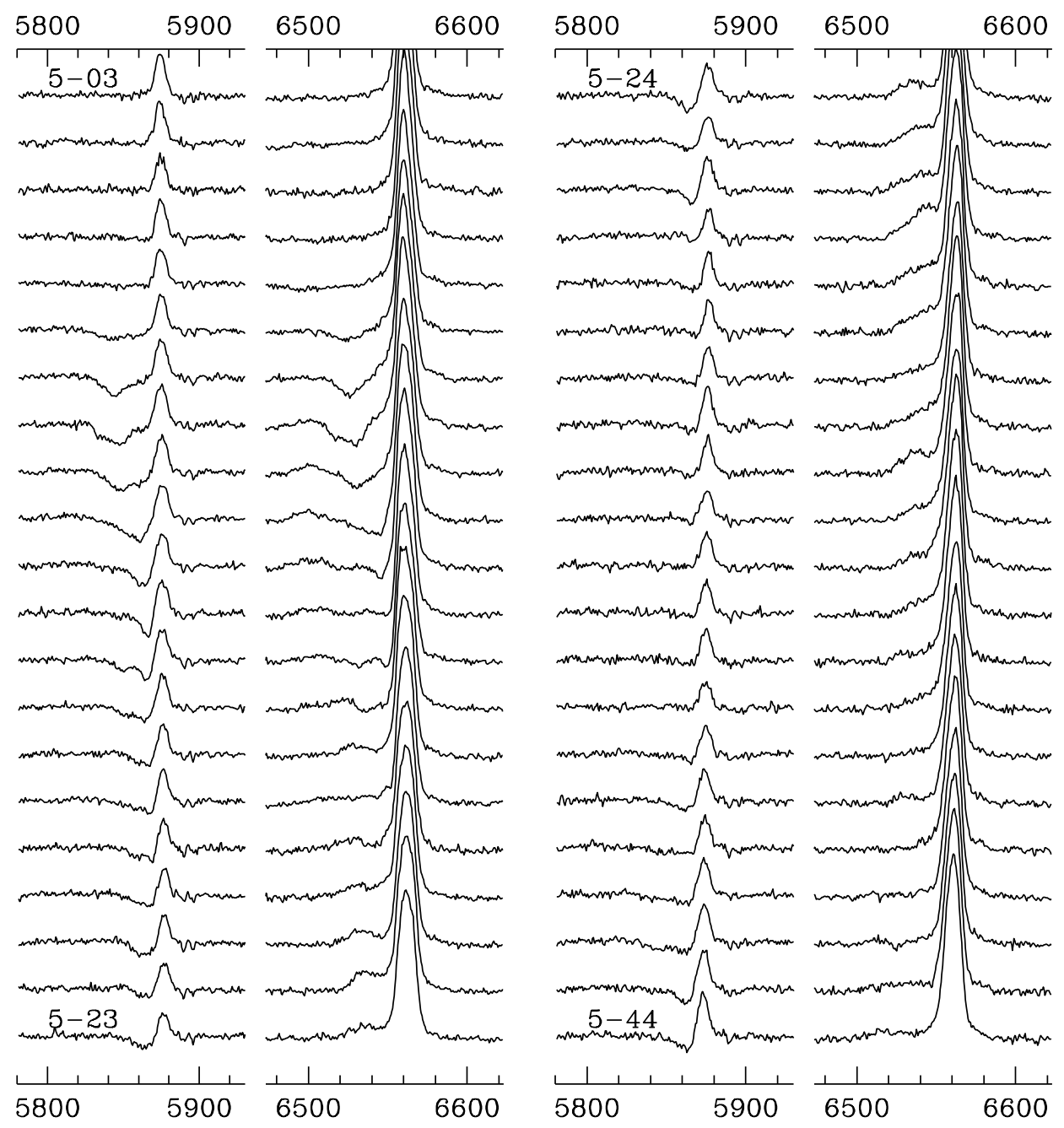

Fig. 2d.- Continuation of the line profile plots. 


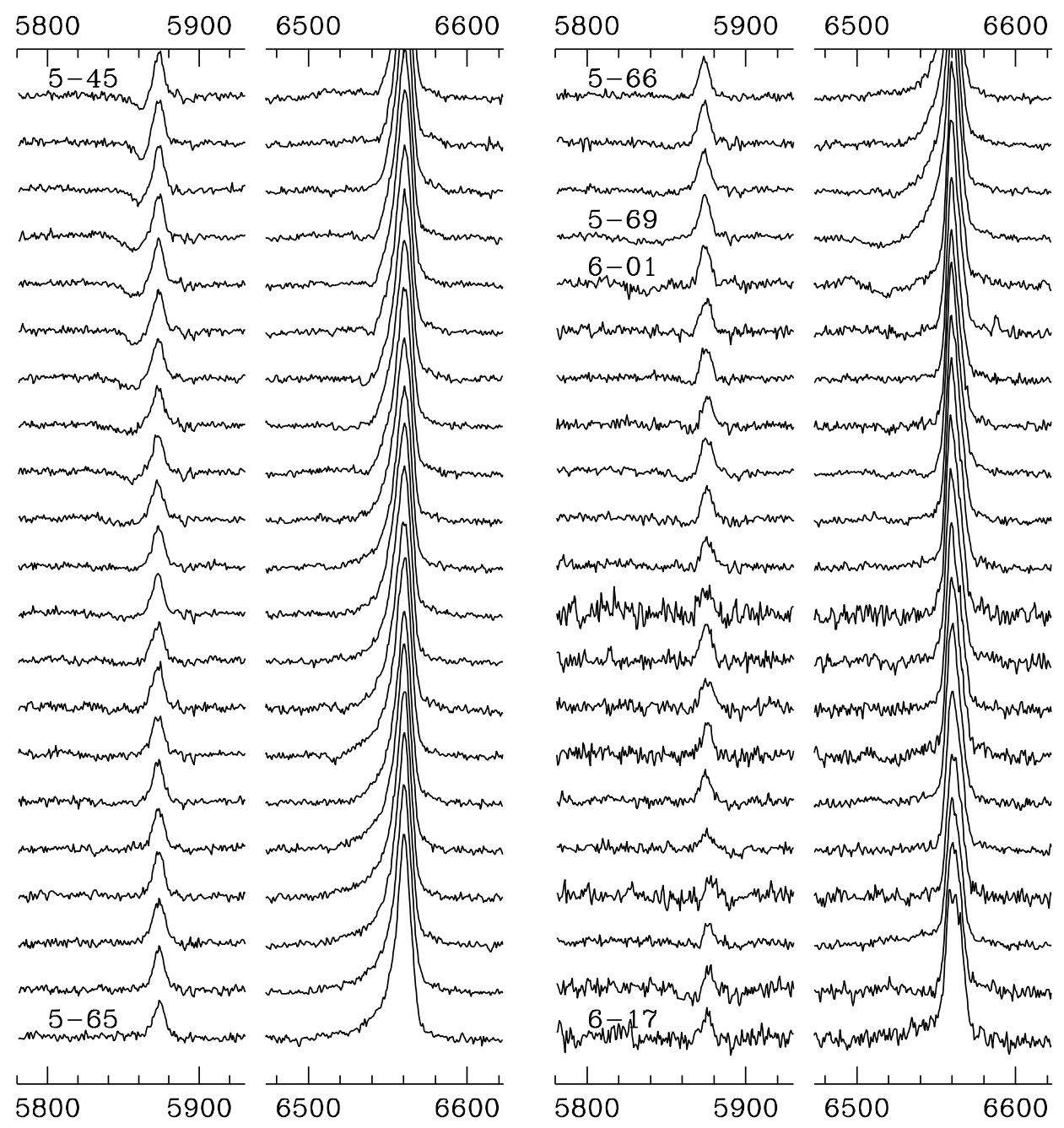

Fig. 2e.- Continuation of the line profile plots. 


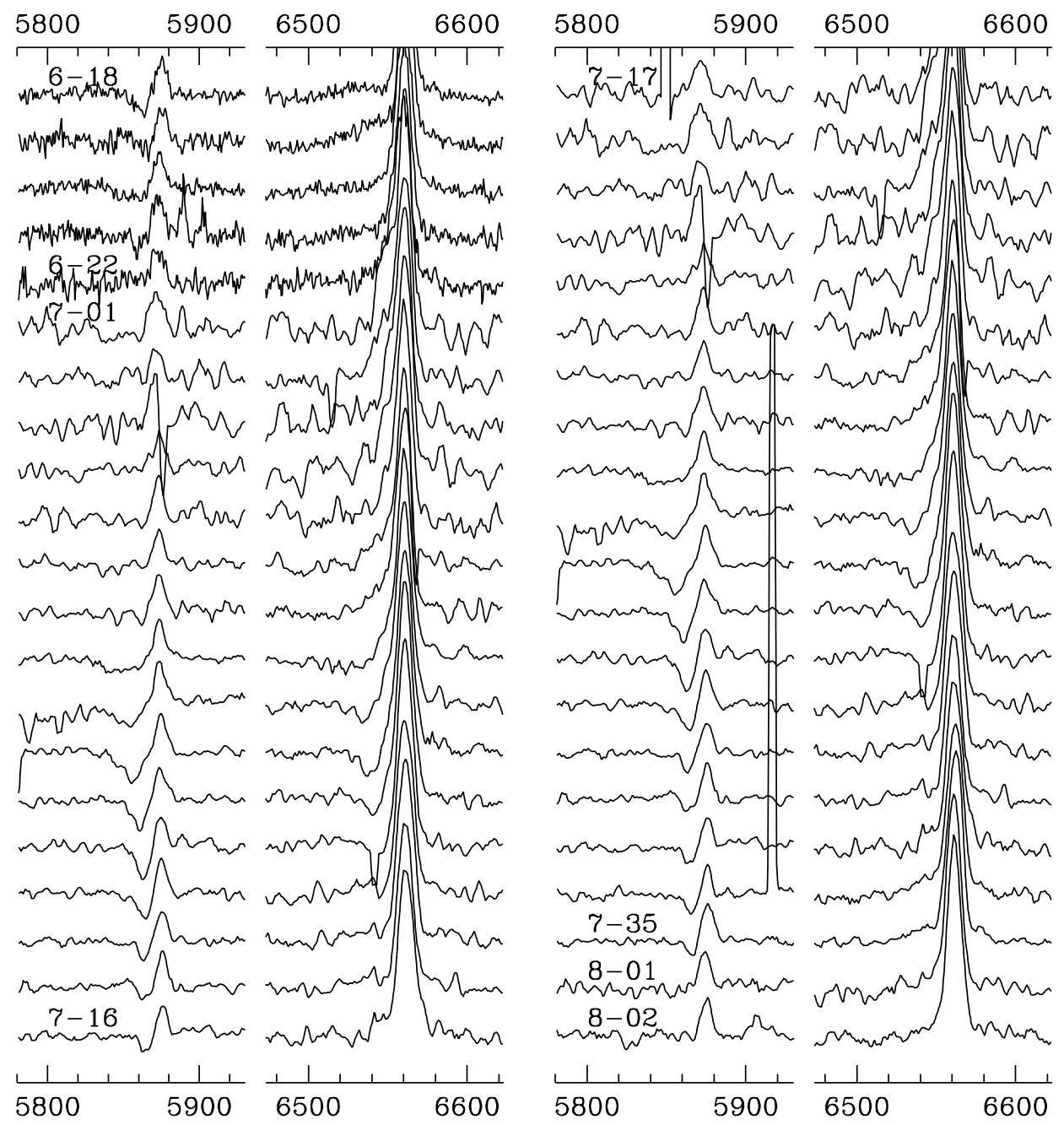

Fig. 2f. - Continuation of the line profile plots. 


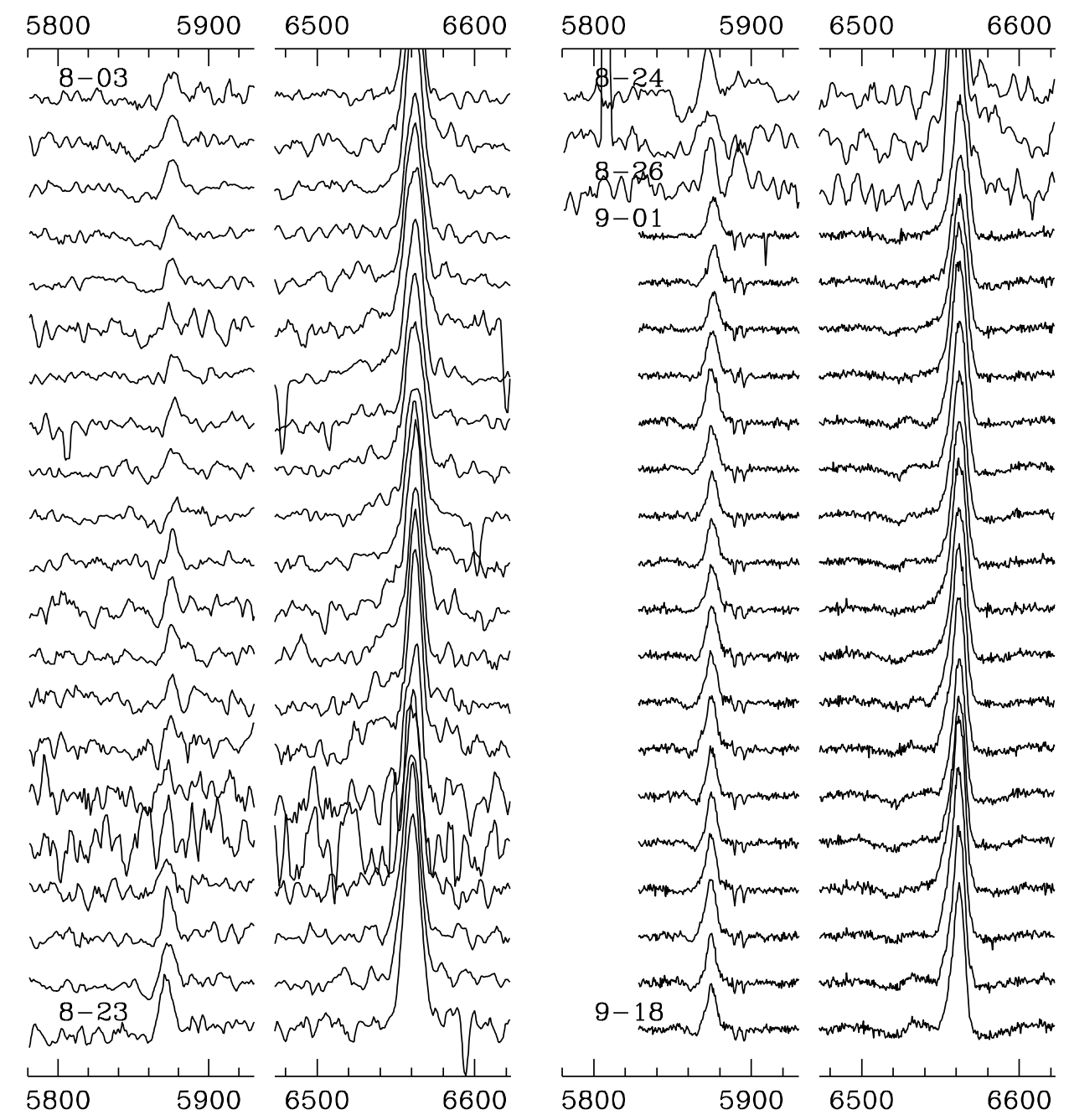

Fig. 2g.- Continuation of the line profile plots. 


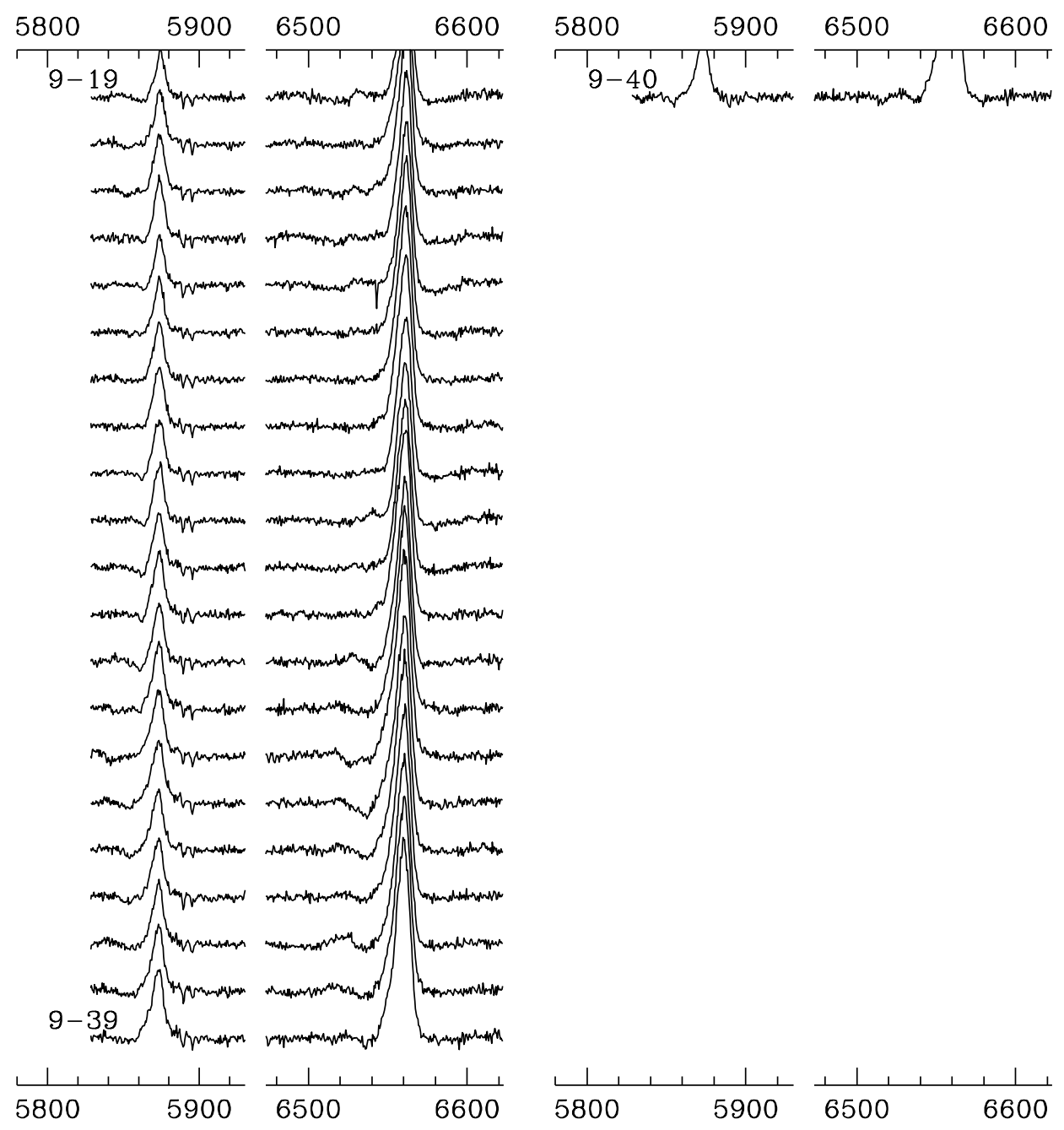

Fig. 2h.- Continuation of the line profile plots. 


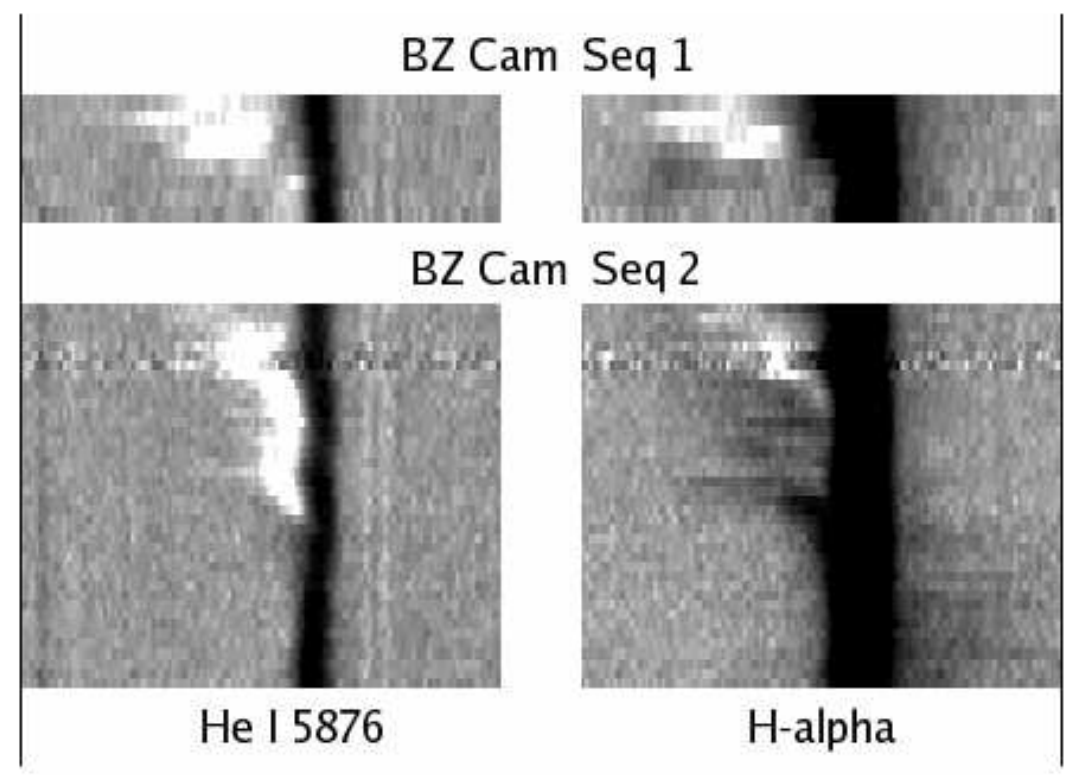

Fig. 3a.- Synthetic trailed spectrum for the data of 2005-Oct-10 UT (top) and 2005-Oct-11 UT (bottom). These spectra are from the KPNO 2.1-m telescope; see Table 3 and the text for details on these two sequences. Black is emission, white is absorption, and time runs down the page. The intensity scales have been enhanced to best show the velocity evolution of wind features. Because the exposure times differ from sequence to sequence, the vertical pixel size has been adjusted so that equal times occupy approximately equal vertical space on these images. Because the wavelength per data point differs from sequence to sequence the data has been interpolated onto a common wavelength scale covering $150 \AA$ around the lines, at $0.5 \AA$ spacing. Some of the sequences were obtained under varying conditions of cloudiness, which sometimes degrades the $\mathrm{S} / \mathrm{N}$ of individual spectra. 


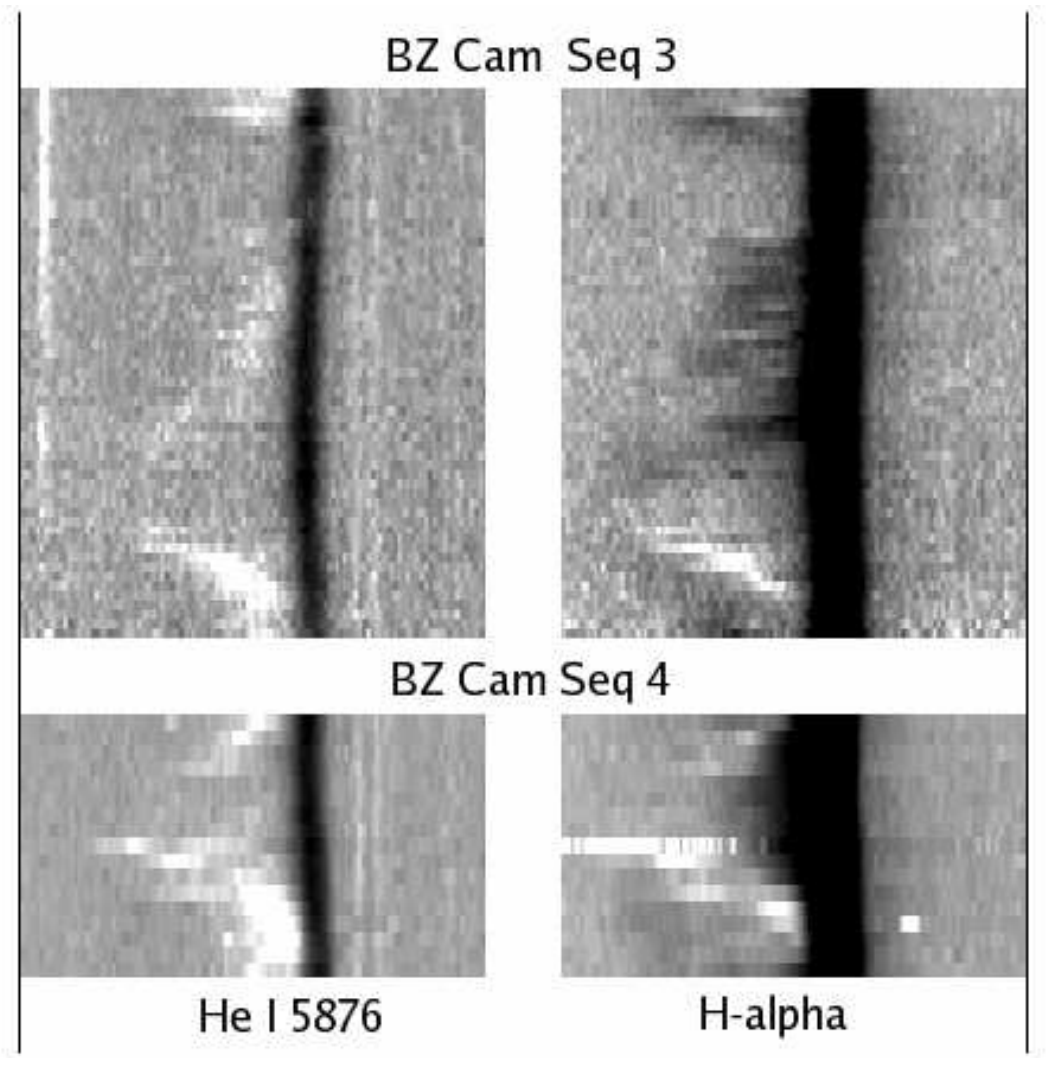

Fig. 3b.- Like Figure 3a except for the data of 2005-Oct-12 UT (top) and 2005-Oct-25 UT (bottom). The top data is from the KPNO 2.1-m telescope, while the bottom data is from the WIYN 3.5-m telescope. The apparent strong blue $\mathrm{H} \alpha$ absorption structure midway in Sequence 4 is an artifact in spectrum 4-9. There is also an artifact just redward of $\mathrm{H} \alpha$ in spectrum 4-24. These features can easily be seen and identified as artifacts in the nested plots of Sequence 4 (Figure 5) 


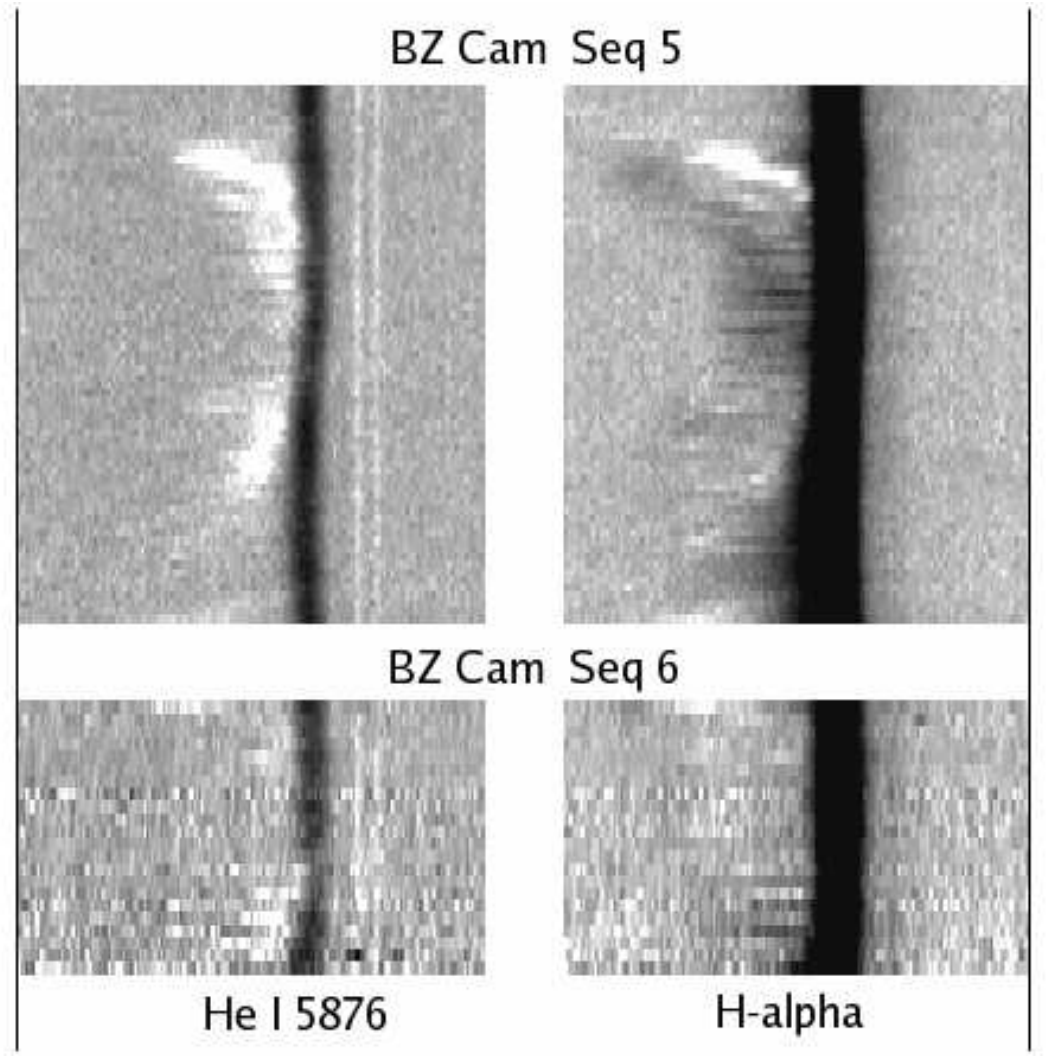

Fig. 3c. - Like Figure 3a except for the data of 2006-Jan-01 UT (top) and 2006-Jan-03 UT (bottom). These data are from the KPNO 4-m telescope. 


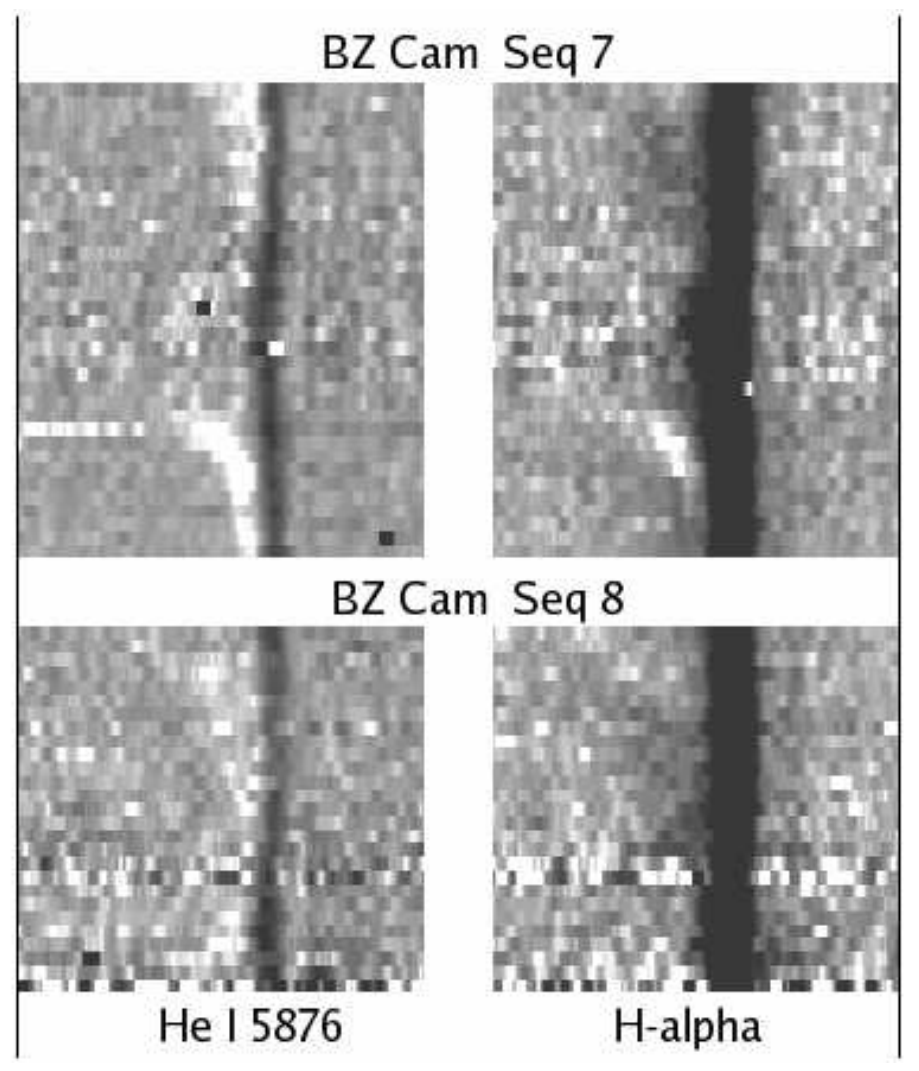

Fig. 3d.- Like Figure 3a except for the data of 2006-Feb-21 UT (top) and 2006-Feb-23 UT (bottom). These data are from the WIYN 3.5-m telescope. Because clouds reduced the S/N of these two sets of WIYN spectra, they have been smoothed with a 3-point triangular filter. 

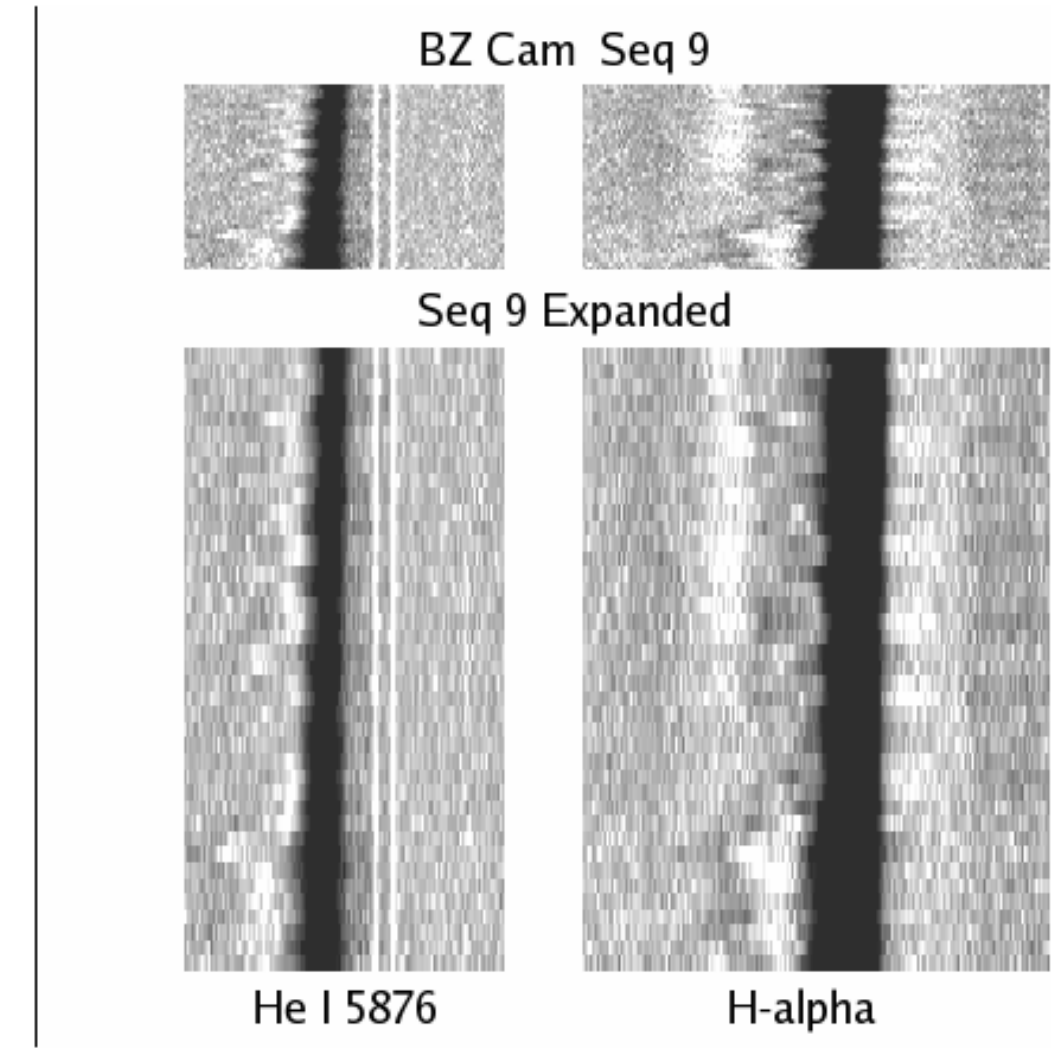

Fig. 3e.- Like Figure 3a except for the data of 2006-Sep-15. These data are from the 6.5-m MMT. The top panel has the same vertical time scale as in Figs 11-14. Because the exposure times for this MMT sequence are quite short, the bottom panel shows the same data, but expanded in the time direction by a factor of 3.3. The region of spectrum to the blue of the He I line is less than in Figs 11-14 because of the blue wavelength cutoff of the original MMT spectra. 


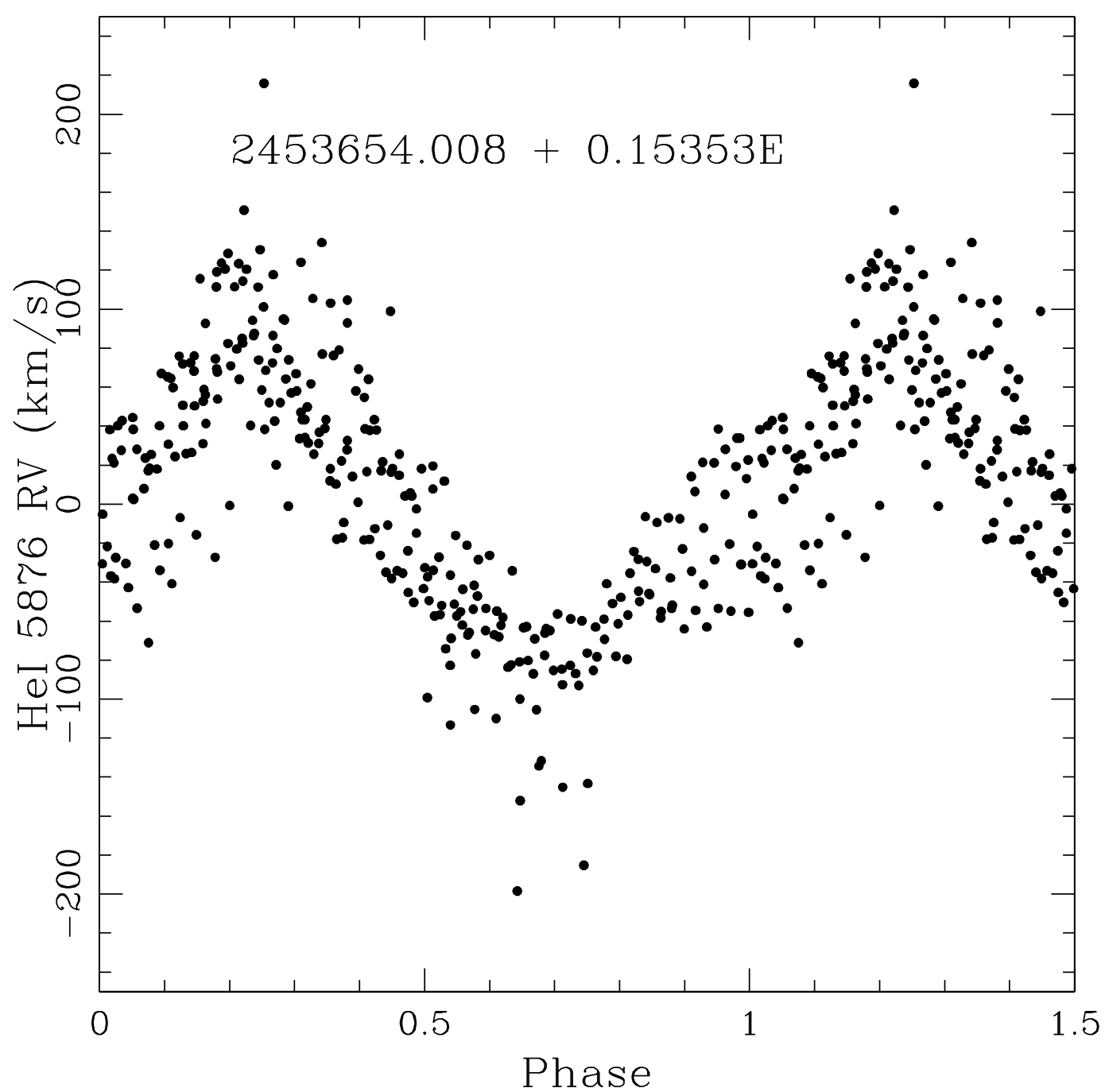

Fig. 4.- The radial velocity curve of the core of HeI 5876, folded on our adopted orbital period. Each night's data was pre-whitened prior to folding. The ephemeris is for the time of - to + crossing of gamma. 


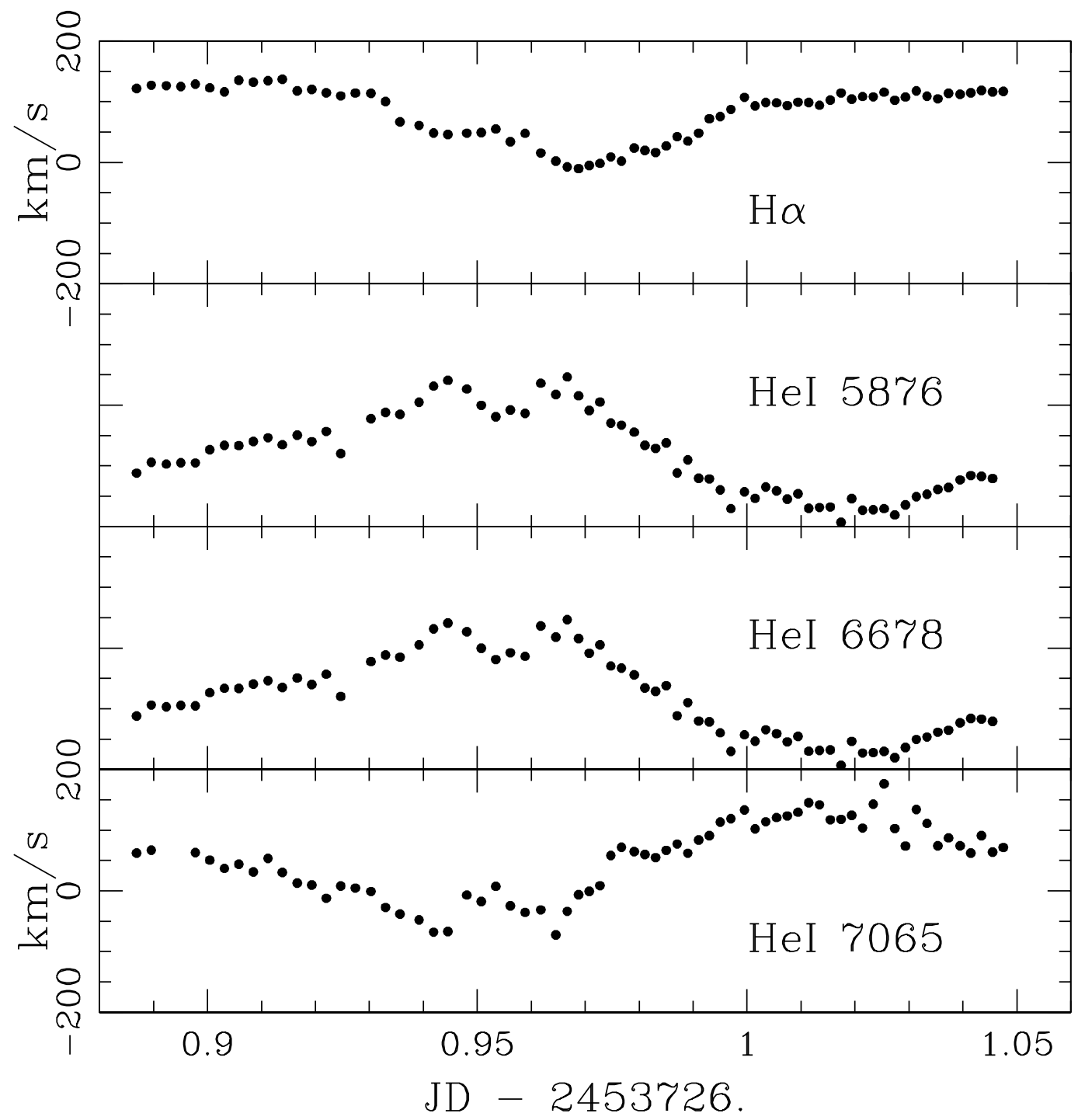

Fig. 5.- The individual radial velocity curves for the cores of 4 emission lines in BZ Cam for the night of 2006-Jan-01 (UT) (Sequence S5 in Table 2) showing the lack of agreement in the shape, amplitude, and phasing of the r.v. curves. These data have not been pre-whitened. See text for details. 


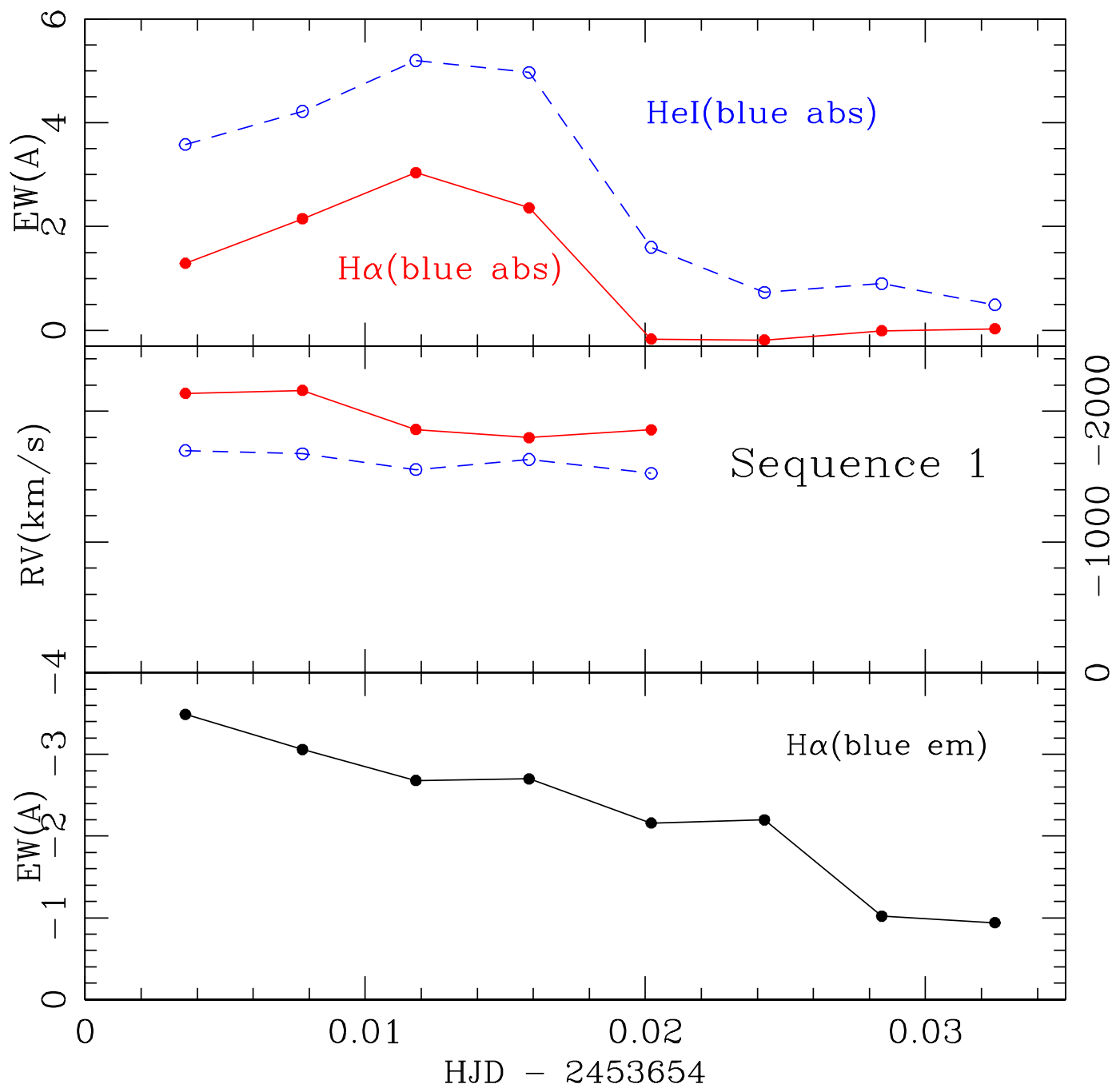

Fig. 6a.- Top panel: Wind strength vs time, as measured by the EW of the P-Cygni absorption in two lines (HeI 5576 (open blue circles)and $\mathrm{H} \alpha$ (filled red circles)), for spectral set S1 (see Table 3). Middle panel: Wind velocity as measured by the radial velocity $(\mathrm{km}$ $\mathrm{s}^{-1}$ ) of the blue absorption, using the same symbol coding as the top panel. Note that the velocity is missing when the feature becomes too weak. Bottom panel: The strength of the blueshifted emission component to $\mathrm{H} \alpha$ which often accompanies a wind event. See text for details on how these quantities are measured. 


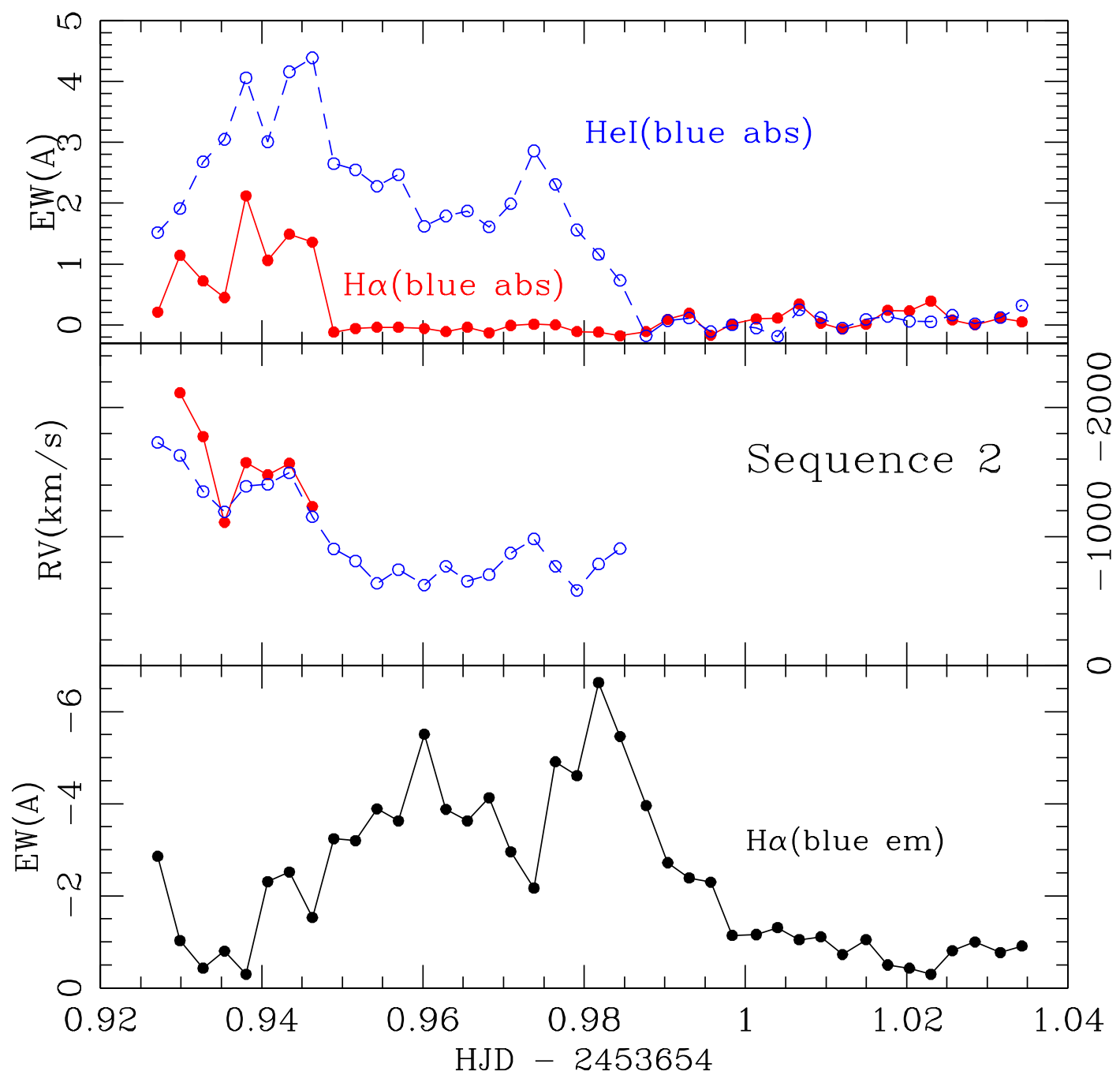

Fig. 6b.- Same as Figure 6a, except for spectral set S2. 


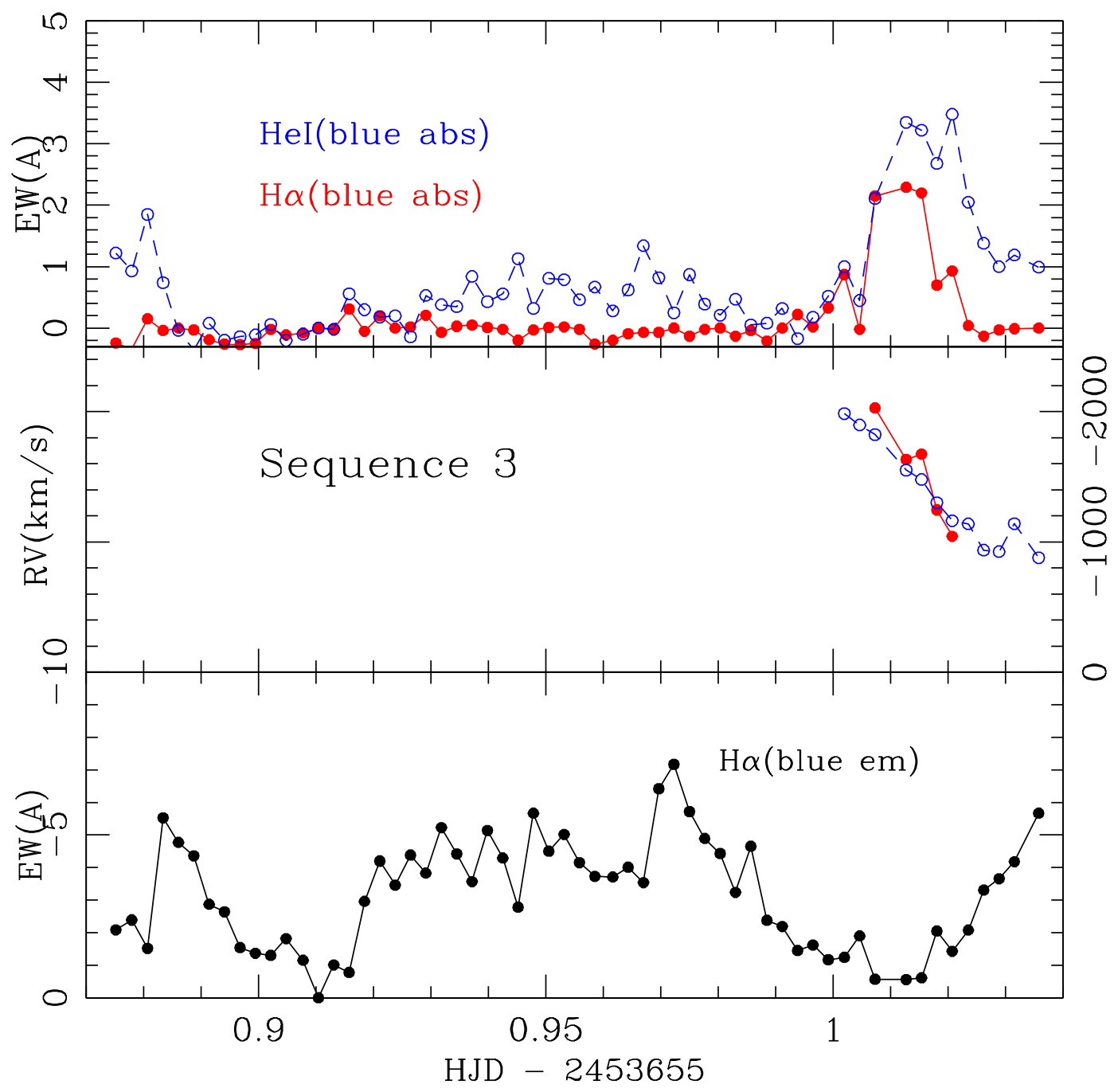

Fig. 6c.- Same as Figure 6a, except for spectral set S3. 


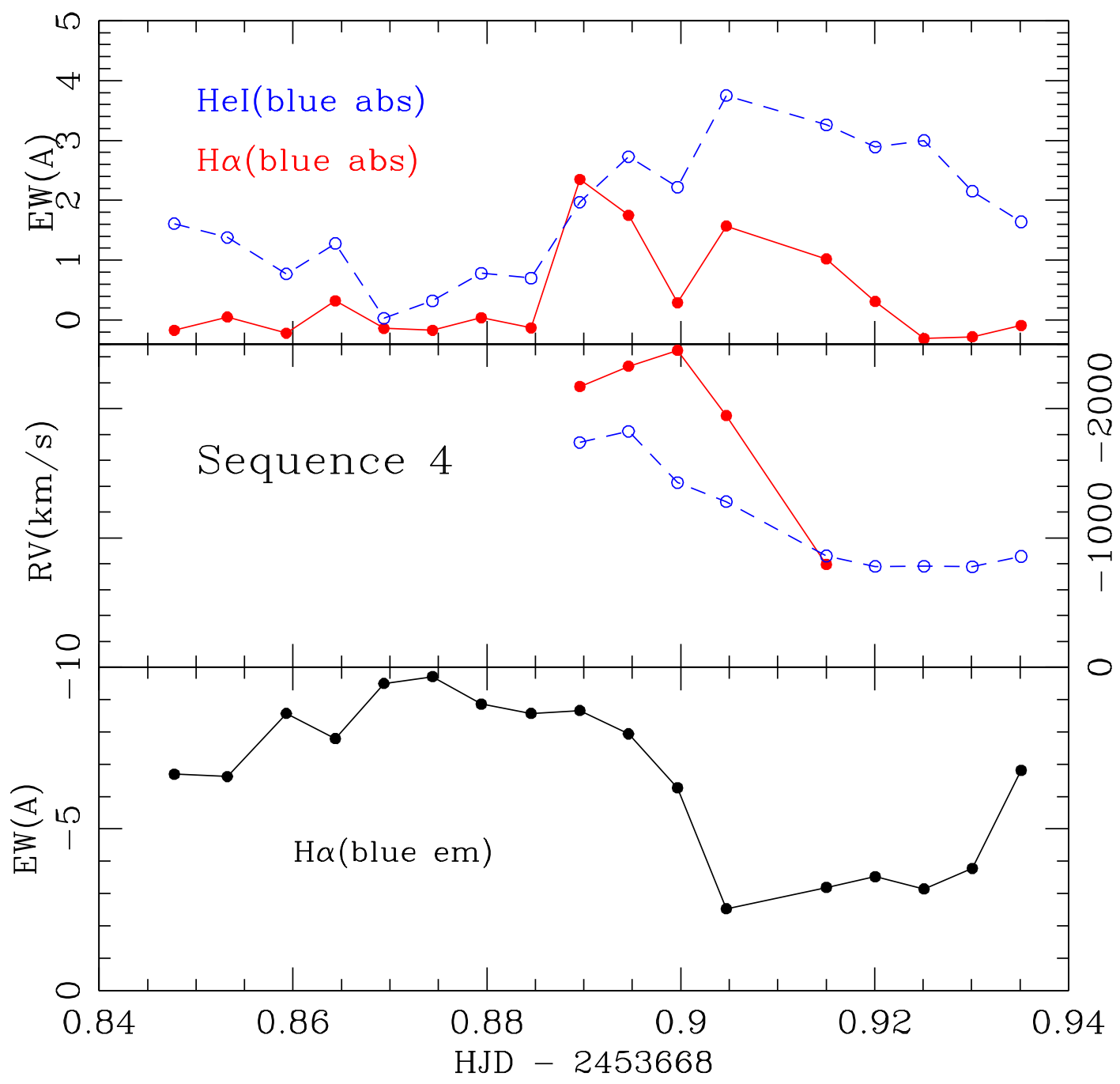

Fig. 6d.- Same as Figure 6a, except for spectral set S4. 


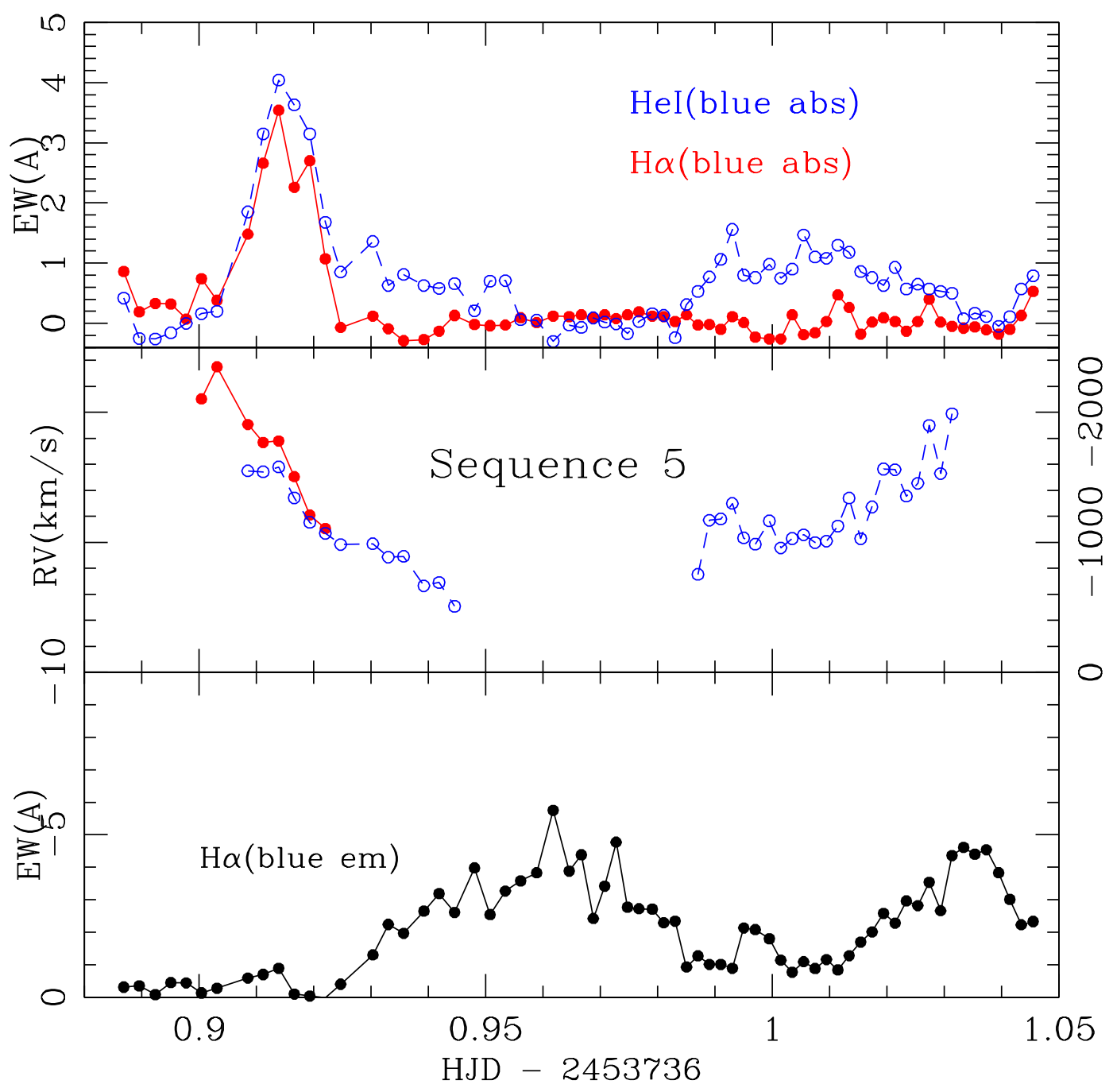

Fig. 6e.- Same as Figure 6a, except for spectral set S5. 


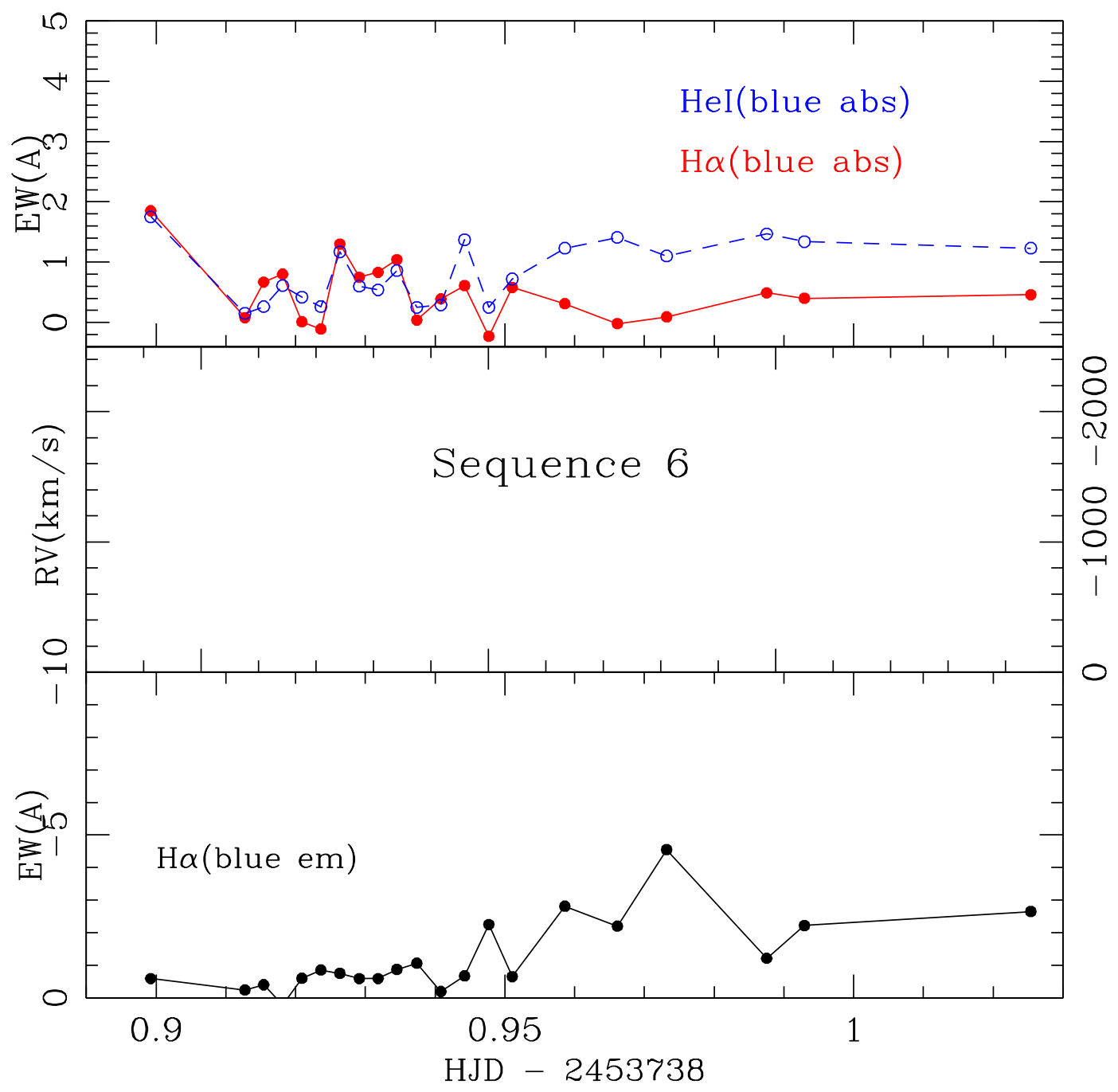

Fig. 6f.- Same as Figure 6a, except for spectral set S6. 


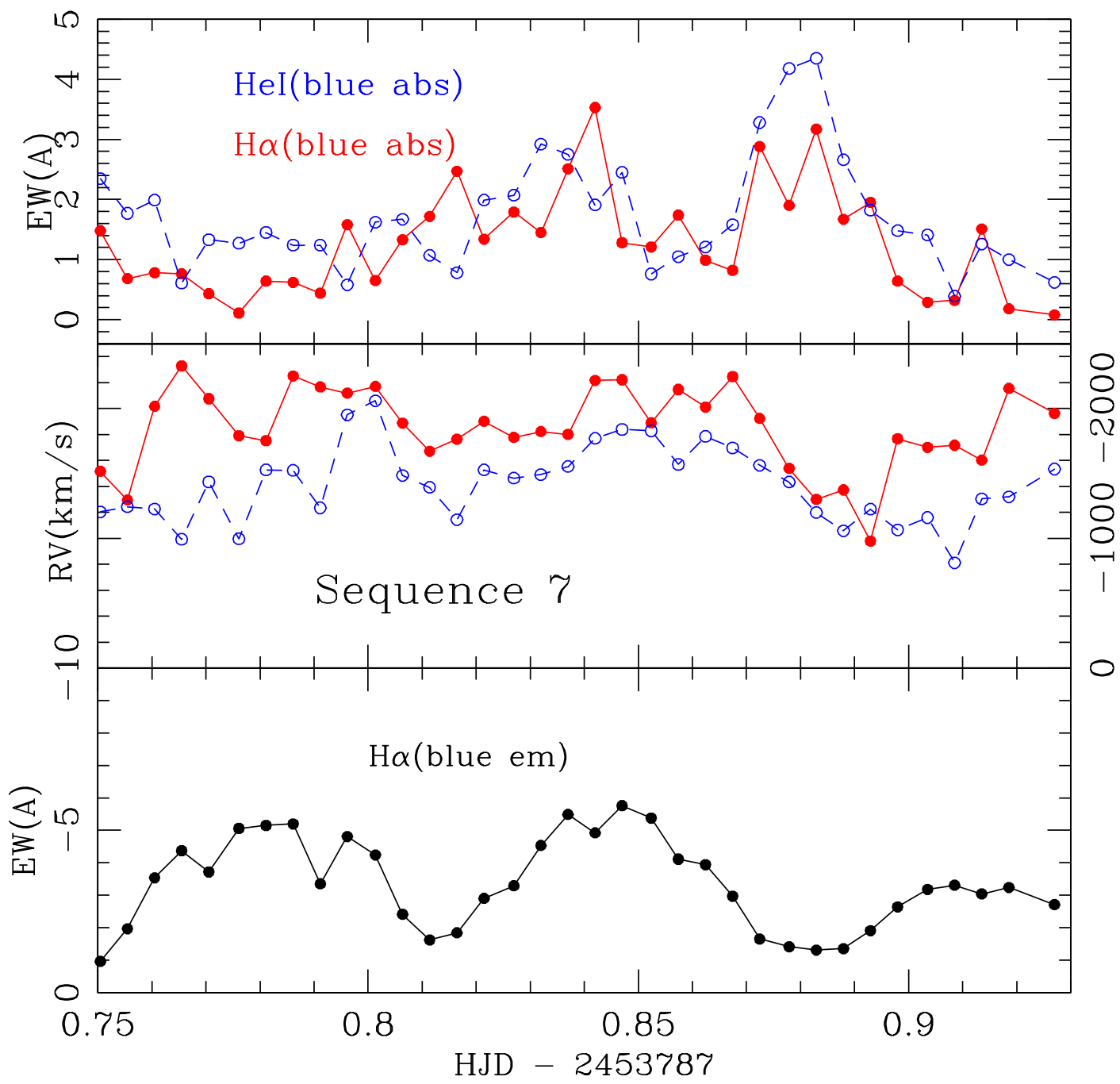

Fig. 6g.- Same as Figure 6a, except for spectral set S7. 


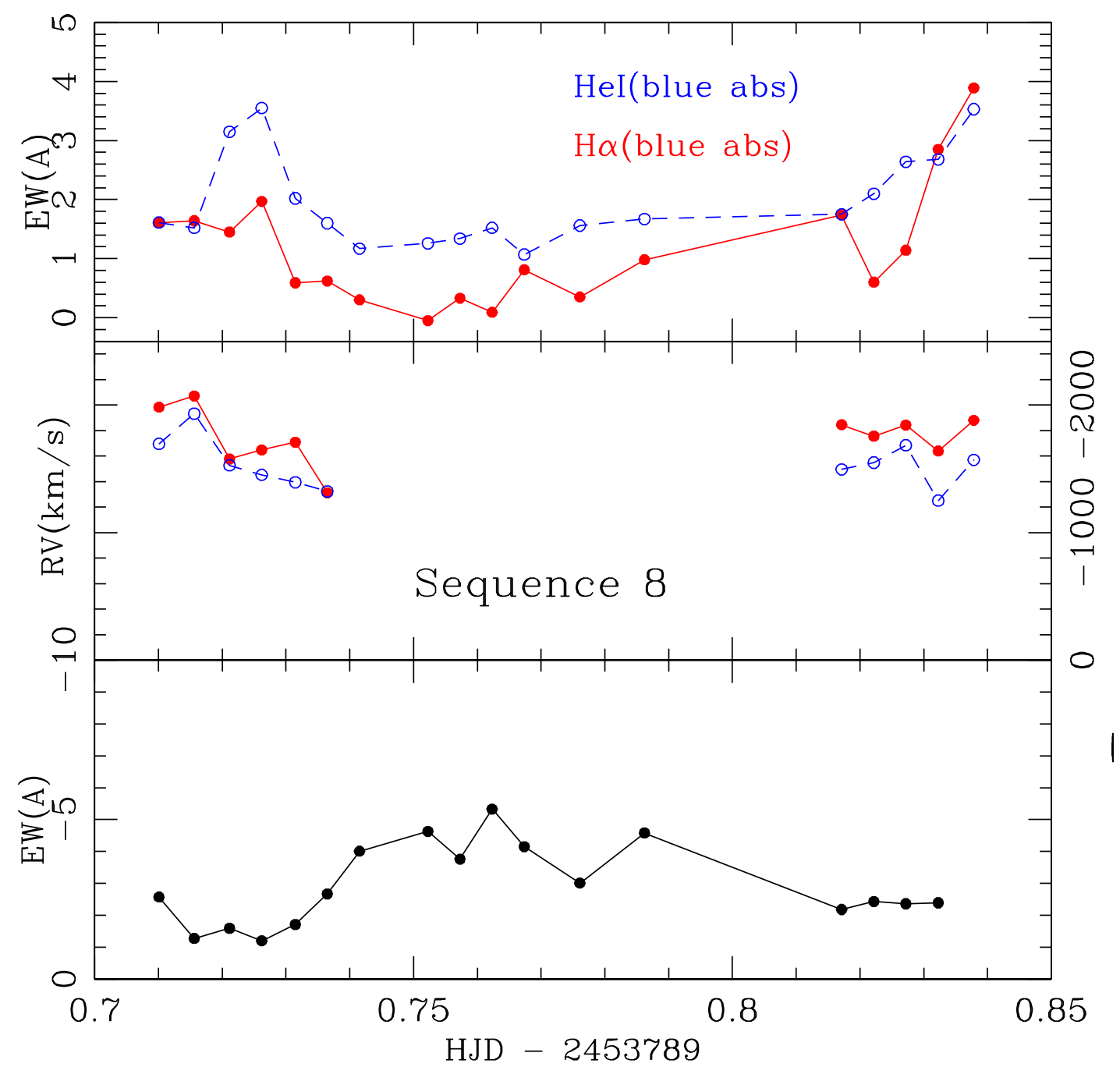

Fig. 6h.- Same as Figure 6a, except for spectral set S8. Comment on lack of data in middle panel. 


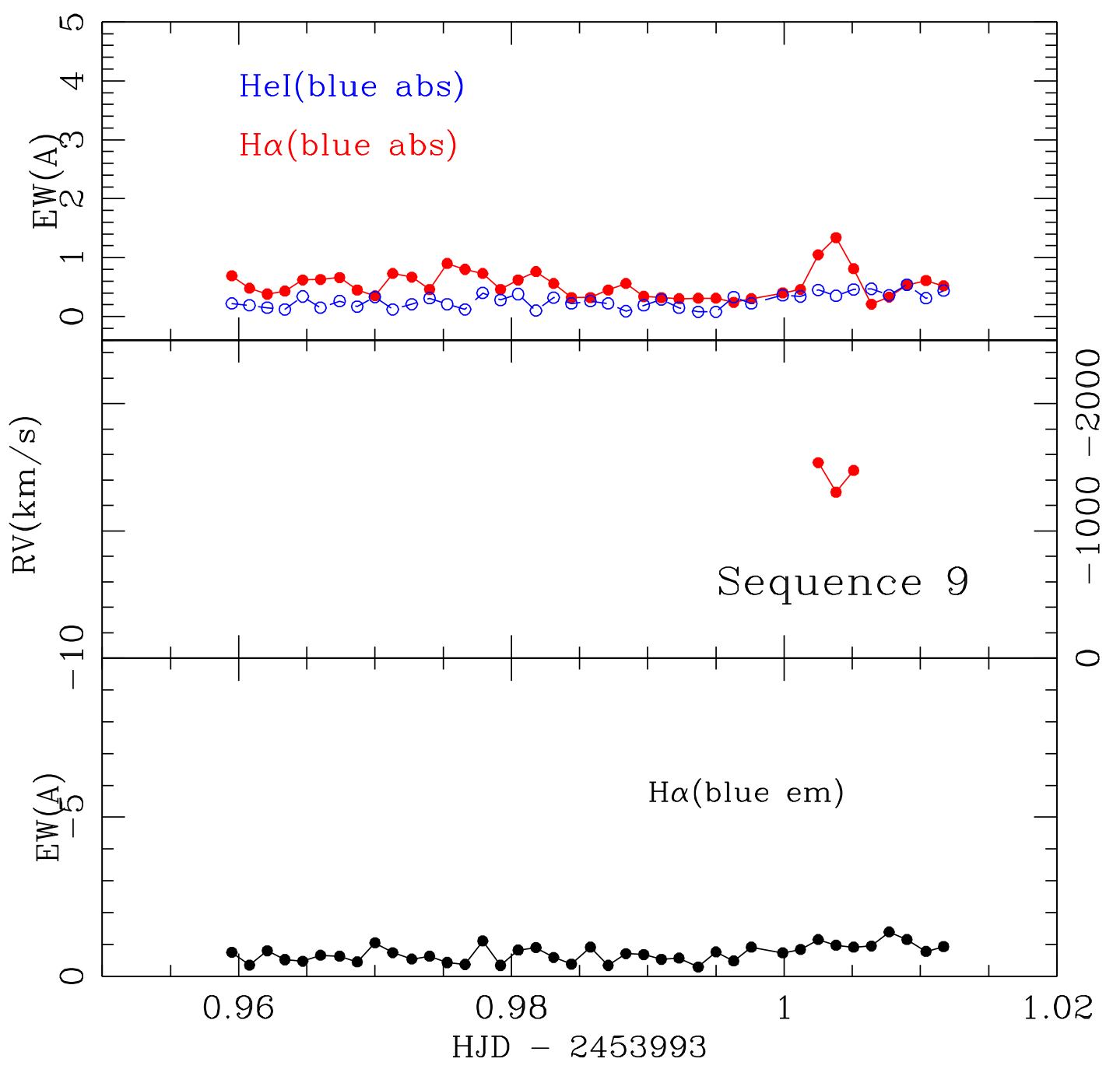

Fig. 6i.- Same as Figure 6a, except for spectral set S9. 


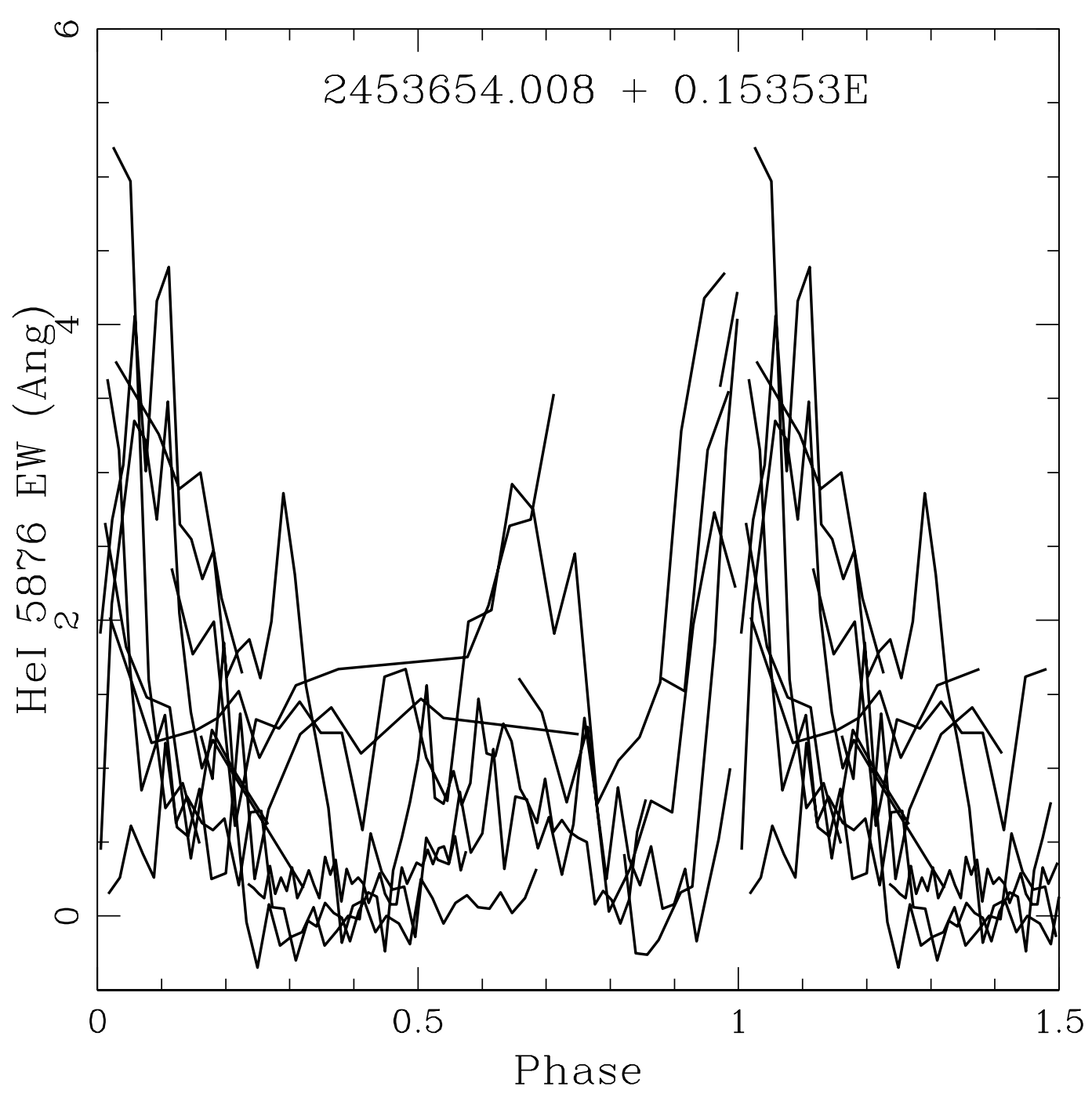

Fig. 7.- The wind strength, as measured by the EW of the blueshifted absorption in HeI 5576, vs orbital phase. All of the EWs from a single spectral set are connected by straight lines. One can see both the episodal nature of individual wind events as well as the concentration of these event to orbital phases just after inferior conjuction of the massgaining star. 

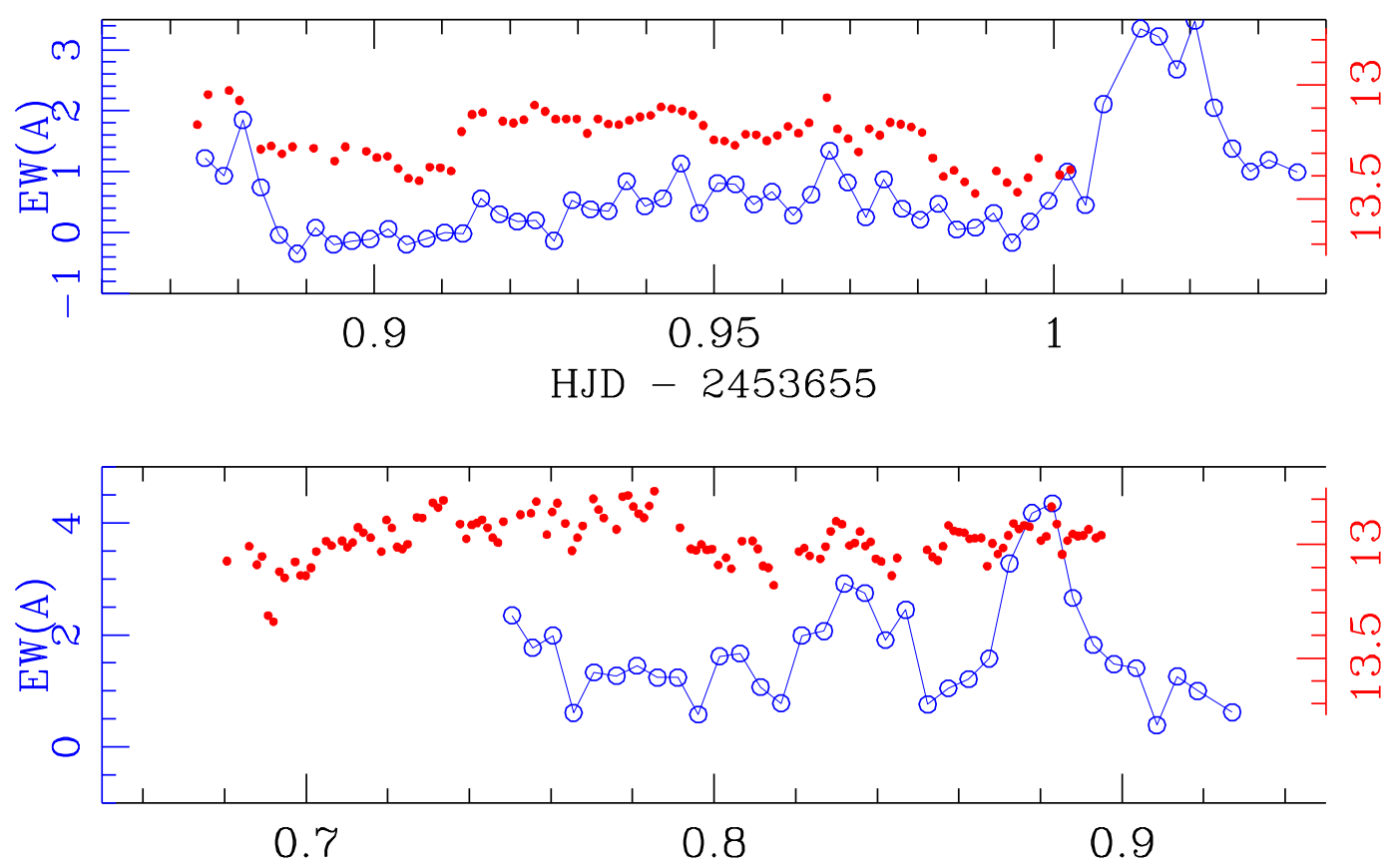

HJD - 2453787

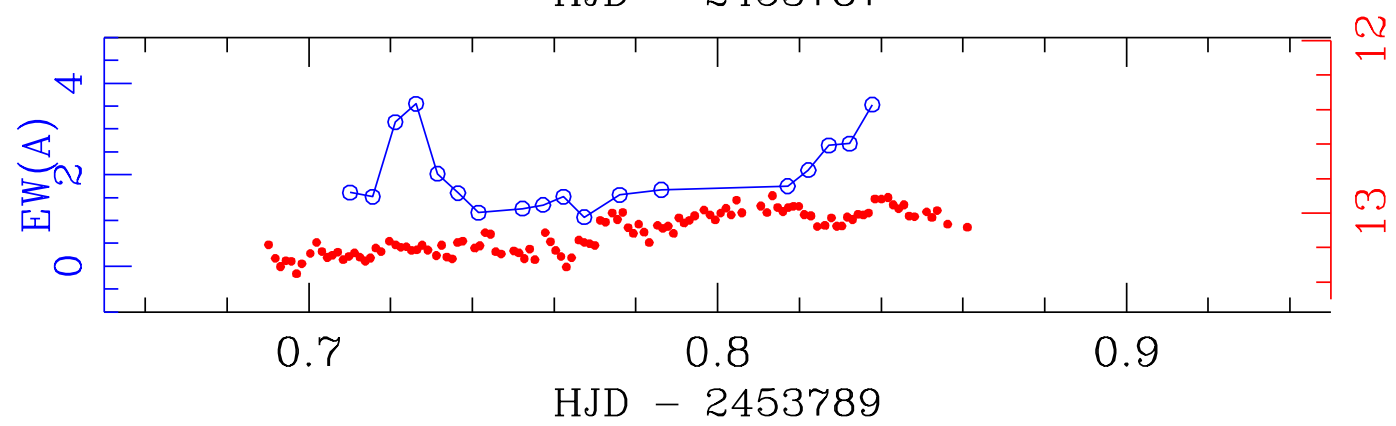

Fig. 8.- Results from simultaneous spectroscopy and photometry on three nights. Top panel: Data from 2005-Oct-12 UT using photometric sequence P4 (closely-spaced small filled circles) and spectroscopic sequence S3 (larger open circles connected by straight lines). The spectroscopy measures the EW of the blueshifted absorption in HeI 5876 (left axis) and the photometry is V magnitude (right axis). Middle panel: Similar data from 2006-Feb-21 using sequences P5 and S7. Bottom panel: Similar data from 2006-Feb-23 using sequences P6 and S8. 